\title{
Algorithmic Foundation and Software Tools for Extracting Shoreline Features from Remote Sensing Imagery and LiDAR Data
}

\author{
Hongxing Liu ${ }^{1}$, Lei Wang ${ }^{2}$, Douglas J. Sherman ${ }^{3}$, Qiusheng Wu ${ }^{1}$, Haibin $\mathrm{Su}^{1}$ \\ ${ }^{1}$ Department of Geography, University of Cincinnati Cincinnati, USA \\ ${ }^{2}$ Department of Geography \& Anthropology Louisiana State University, Baton Rouge, USA \\ ${ }^{3}$ Department of Geography, Texas A\&M University College Station, USA \\ E-mail: Hongxing.Liu@uc.edu, leiwang@lsu.edu, sherman@geog.tamu.edu, \{wuqe,suhn\}@mail.uc.edu \\ Received January 3, 2011; revised January 8, 2011; accepted January 12, 2011
}

\begin{abstract}
This paper presents algorithmic components and corresponding software routines for extracting shoreline features from remote sensing imagery and LiDAR data. Conceptually, shoreline features are treated as boundary lines between land objects and water objects. Numerical algorithms have been identified and devised to segment and classify remote sensing imagery and LiDAR data into land and water pixels, to form and enhance land and water objects, and to trace and vectorize the boundaries between land and water objects as shoreline features. A contouring routine is developed as an alternative method for extracting shoreline features from LiDAR data. While most of numerical algorithms are implemented using $\mathrm{C}++$ programming language, some algorithms use available functions of ArcObjects in ArcGIS. Based on VB .NET and ArcObjects programming, a graphical user's interface has been developed to integrate and organize shoreline extraction routines into a software package. This product represents the first comprehensive software tool dedicated for extracting shorelines from remotely sensed data. Radarsat SAR image, QuickBird multispectral image, and airborne LiDAR data have been used to demonstrate how these software routines can be utilized and combined to extract shoreline features from different types of input data sources: panchromatic or single band imagery, color or multi-spectral image, and LiDAR elevation data. Our software package is freely available for the public through the internet.
\end{abstract}

Keywords: Shoreline Extraction, Remote Sensing Imagery, LiDAR Data, ArcGIS, ArcObjects, VB.NET

\section{Introduction}

A shoreline is a spatially continuous line of contact between the land and a body of water (sea or lake). The terms "shoreline" and "coastline" are often interchangeably used in geosciences and coastal research communities [1]. It has been long recognized that information about shoreline position, orientation, and geometric shape is essential for coastal scientists, engineers and managers. Depending on the application context, the requirements for shoreline information vary in terms of shoreline positional accuracy, spatial resolution and coverage, and temporal frequency in survey and mapping. In the design of shipping structures, coastal defense and protection infrastructure, coastal engineers often need the precise geographical position and detailed shape of sho- relines within a certain coastal stretch [2]. Coastal managers and land use planners rely on up-to-date shoreline information at regional scales for establishing legal property boundary definition and building setback lines [3,4], estimating recreational beach width and volume [5], inventorying wetland and agricultural land resources [6,7], delineating flood and hurricane hazard zones, and assessing the coastal vulnerability and response management strategies to climate changes [8-10]. Coastal scientists and geomorphologists have utilized multi-temporal shoreline data for estimating sediment transport and budgets [11], examining coastal erosion and accretion [12,13], quantifying historical shoreline retreating or advancing rates $[12,14,15]$, and assessing sea level rise and its impacts [16,17].

Traditionally, shorelines depicted on nautical charts 
and topographic maps were compiled through visual interpretation of aerial photographs [18]. High resolution aerial photographs contain details of terrain features, and shorelines can be observed and delineated with great precision. In recent decades, new approaches have been developed for shoreline mapping, including the use of high-resolution satellite imagery [19], and airborne LiDAR technology [20-22]. The era of 1-meter or submeter satellite imagery such as IKONOS, QuickBird, WorldView and GeoEye, present new and exciting opportunities for geosciences and coastal research community. The advent of airborne LiDAR technology offers an alternative means for mapping coastal topography and shoreline features with unprecedented accuracy and flexibility. When the LiDAR data are acquired at a low water level, it is possible to derive various shoreline indicators with reference to different tidal datums [22,23]. This represents a major advantage of the LiDAR data over the remote sensing imagery.

One important technical challenge for shoreline mapping is to accurately and efficiently interpret and extract shoreline features from remote sensing imagery and LiDAR data onto a vector representation in a map format. Traditionally, shorelines were manually delineated with a pencil on vellum paper overlaid on top of aerial photographs or traced with a cursor from digital remote sensing images on the computer screen. The manual tracing method is tedious, subjective, time-consuming, and labor intensive, contributing to long periods between successive shoreline maps. In the past decades, a great deal of research effort has been devoted to the automation of shoreline extraction from remote sensing data. Lee and Jurkevich [24] presented an edge detection algorithm for extracting shorelines from a satellite Synthetic Aperture Radar (SAR) image. Ryan et al. [25] proposed an image segmentation approach to the shoreline extraction problem and tested their method on scanned USGS aerial photographs. Mason and Davenport [26] employed an edge detection method with a coarse-fine resolution processing strategy and applied their approach to satellite SAR images. Liu and Jezek [27,28] developed an automated shoreline extraction method based on a locally adaptive thresholding algorithm, and its effectiveness has been demonstrated with both optical and radar images. In recent years, numerical techniques have also been developed to process LiDAR data for extraction of shorelines. Stockdon et al. [29] determined the shoreline position by fitting regression lines on cross-shore LiDAR elevation profiles. The contouring method has been used by many researchers to derive shorelines from the LiDAR data [21,30,31]. Liu et al. [22] developed a segmentation-based method for extracting tidal datum referenced shorelines from LiDAR data. Despite the pro- gresses described above, very few studies have been conducted for a comprehensive analysis and assessment of algorithmic foundation for extracting shoreline features from both remote sensing imagery and LiDAR elevation data. To our knowledge, no dedicated software tools exist at present for automated shoreline extraction.

Based on our previous research and application experiences [22,23,27,28,32], we made a critical assessment of existing algorithms for shoreline extraction. This paper aims to identify the best algorithmic components for shoreline extraction and to present methods for implementing these algorithms into re-usable software routines. The algorithms and software routines presented in this paper are object-based in the sense that shoreline features are treated as boundary lines between land objects and water objects. Numerical algorithms are devised and combined to create continuous land and water objects and then trace the boundaries between land and water objects into vector shoreline representations. Further, an optimized contouring routine is developed as an alternative approach to shoreline delineation from LiDAR data. The software routines and the graphical user's interfaces are implemented using the object-oriented programming (OOP) languages $\mathrm{C}++$ and VB.NET in conjunction with ESRI ArcObjects. While most core numerical algorithms are implemented using the computationally high performance $\mathrm{C}++$ programming language, some are realized using available functions of ArcObjects in the ArcGIS environment. A graphical user's interface has been developed for each routine by programming ArcObjects through VB.NET language. Consequently, the software has been integrated as an ArcGIS extension module named as "ShorelineExtractor". In this paper, Radarsat SAR image, QuickBird multispectral image, and airborne LiDAR data have been used to illustrate how software routines can be utilized and combined to automate shoreline extraction from different data sources: panchromatic or single band imagery, color or multi-spectral image, and LiDAR elevation data. We believe that this paper and the corresponding software package will provide the geosciences and coastal research community with a powerful tool for efficiently processing high-resolution remote sensing imagery and LiDAR for frequent and timely shoreline measurements.

\section{Algorithmic Foundations for Shoreline Extraction}

Shoreline extraction from imagery is a complicated process. Because of frequent lack of sufficient contrast between the land and water bodies on images and the difficulty in distinguishing shoreline features from other linear features, most general-purpose algorithms for edge 
and linear feature detection are not adequate for the automated coastline extraction. Previously, two approaches have been employed for shoreline extraction from images. One approach treats shoreline extraction as an edge detection problem. This approach is based on the observation that image intensity (gray) values across the shoreline change abruptly, so that spatial differentiation operators and edge detectors may be used to locate meaningful intensity variations and discontinuities on the image as candidate shoreline pixels. The other approach treats the shoreline as the boundary between two relatively homogeneous objects (land and water objects), each with a distinct average intensity value. The edge-based approach suffers from the fact that the edge pixels produced by edge detectors are often discontinuous and seldom characterizes a shoreline completely $[24,26,28]$. To assemble and link edge pixels separated by small breaks is a computationally intensive task, even for a crude approximation [24,33]. In contrast, the object-based approach has the advantage of creating a continuous boundary between the land and water objects $[23,28]$. Therefore, we adopt the object-based approach for the development of shoreline extraction algorithms and software routines for image data.

Different from remote sensing imagery, the LiDAR data are elevation measurements rather than the reflected intensity of radiation. The shorelines cannot be visually interpreted from LiDAR data, but can be inferred from the elevation values with reference to a tidal datum. We adopt two approaches to the processing of LiDAR data for shoreline extraction. The first is object-based, similar to the one used for processing image data. By comparing the LiDAR DEM with the tidal datum surface, grid cells/pixels with an elevation value higher than the tidal datum can be grouped into land objects, while grid cells/pixels with an elevation below the tidal datum can be grouped into water objects. The second approach is contouring-based. The idea is that after the LiDAR elevation values are adjusted with reference to a tidal datum, the grid cells/pixels on the shoreline should assume a value of zero. Thus, the shorelines can be derived by contouring zero elevation pixels.

As indicated above, the object-based approach is applicable to both remote sensing image data and LiDAR elevation data. This approach is implemented with four groups of algorithms and software routines: preprocessing, land/water segmentation, object post-processing, and shoreline generalization. With the object-based approach, algorithms and software routines used for processing image data and LiDAR data are almost the same, except for those used for land/water segmentation. The preprocessing algorithms aim to suppress data noise and enhance the contrast between land and water bodies. The segmentation algorithms partition an image into homogeneous land and water objects. The post-object processing algorithms are designed to differentiate the shoreline features from other linear features, and trace the shoreline pixels into a vector representation. The contouring based approach is only applicable to LiDAR data. This approach consists of three groups of algorithms and software routines: preprocessing, shoreline contouring with a specified reference tidal datum, and shoreline generalization. Our goal is to create an effective, operational software tool that minimizes operator's intervention and editing efforts and maximizes the reliability, repeatability, and accuracy of the derived shoreline features.

We have identified a sequence of key algorithm elements and implemented 14 software routines to automate the coastline extraction process (Figure 1). The shoreline extraction process can be decomposed into a sequence of processing tasks. The combination of these routines is capable of processing panchromatic/single band images, color/multi-spectral band images, and LiDAR elevation data for shoreline extraction. Each application scenario requires a different set of routines as described in the following section. We implemented the key algorithms as a series of re-usable DLLs (Dynamic-Link Library) using the computationally high performance language C++.NET. These re-usable algorithm components (DLLs) can be easily incorporated into commercial or open source GIS and remote sensing software packages as a plug-in component, including the open source GIS software-GRASS, the proprietary GIS software-ArcGIS, and the proprietary remote sensing software-ENVI.

Considering the widespread use of ArcGIS software packages in geosciences and coastal research communities, in this research we select to seamlessly embed our shoreline extraction software routines into ArcGIS as an extension module. The combination of the specialized shoreline extraction programs with ArcGIS as a single integrated software package allows users to take advantage of the powerful ArcGIS functions in data management, visualization, and spatial analysis during the shoreline extraction process. In this way, the input images and LiDAR elevation data in ArcGIS compatible format can be directly loaded in the shoreline extraction module. Intermediate processing results can be immediately displayed and checked by using the variety of ArcGIS visualization functions. The shorelines extracted from remote sensing data are output in ArcGIS data formats and can be further edited and validated using the ArcGIS's editing capabilities. Using ArcGIS spatial analysis functions, final shoreline products can be readily compared and integrated with other data layers for map composition and change analysis. 


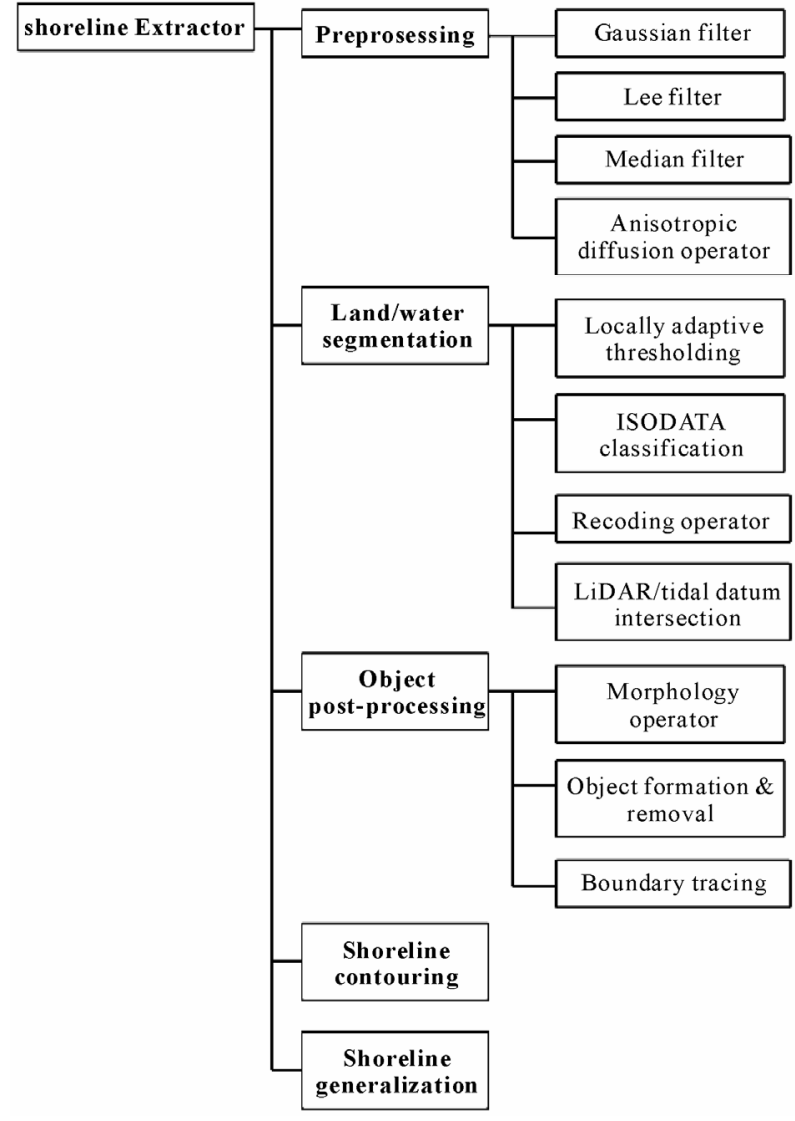

Figure 1. Structure and software routines for shoreline extraction.

We constructed the shoreline extraction extension module using ArcObjects, which are a set of platform independent software components designed by ESRI Inc. specifically for developing ArcGIS applications. We choose VB.NET to program ArcObjects for creating the shoreline extraction extension module. The use of VB.NET makes it easier to package and deploy the extension module. In addition, it is possible to make the extension module as a standalone application running outside ArcGIS using ESRI's map control. Our extension module can be wrapped and distributed as a single package and installed with a user friendly setup procedure. After installation, the shoreline extraction extension module appears in ArcGIS as shown in Figure 2.

For each software routine, we devise its graphical interface through a VB.NET program that calls and wraps the relevant ArcObjects and our specialized DLLs developed using $\mathrm{C}++$ language. The graphical interface for each routine is a customized dialogue menu that guides the user to load the input data, to set the relevant parameter values, and to specify the outputs. This eliminates the needs for the user to remember the command syntax and relevant parameter values.

\section{Algorithm Components and Software Routines}

Our shoreline module is capable of handling various types of remote sensing images and LiDAR elevation data. The commonly used remote sensing image sources for shoreline mapping include panchromatic aerial photographs, natural color aerial photographs, near infrared color aerial photographs, panchromatic satellite images, multi-spectral satellite images, and synthetic aperture radar (SAR) images. The input images need to be georeferenced and orthorectified before the shoreline extraction operation. The LiDAR data need to be prepared in a raster grid format and be vertically referenced to a tidal datum surface in order to derive tide coordinated shoreline. In the following sections, we describe algorithms and routines implemented in our extension module, with the emphasis on the technical improvements.

\subsection{Algorithms and Routines for Preprocessing}

The purpose for preprocessing the images and LiDAR data is to reduce data noise and enhance the contrast between land and water masses for shoreline edge detection. The noise in images and LiDAR data can result in numerous isolated, small, insignificant or spurious edges other than real shoreline features. This imposes great complications in subsequent image processing. To address the noise issue, we select and implement several specific noise-reduction filters from the filters published in the literature. For the shoreline extraction purpose, the filters used in the preprocessing stage should have an edge-preserving property, namely, the ability to remove data noise while preserving the precise position of shoreline edges. We select and implement four of such filters for handling different types of input data: Gaussian filter, Lee Sigma filter, median filter, and anisotropic diffusion operator. It should be noted that although the mean (average) filter is widely available, it should not be used in the shoreline extraction process because the use of the mean filter blurs the land-water boundary edges and increases the shoreline positional error.

1) Gaussian filter: it uses a weight kernel that represents the shape of a Gaussian (bell-shaped) hump and outputs a weighted average of pixels in the kernel neighborhood, with a higher weight assigned to pixels closer to the central pixel. The Gaussian filter provides gentle smoothing of data noise without seriously blurring of major edge features. We implement the Gaussian filter through a VB.NET program to call ArcObjects and invoke $\mathrm{C}++$ programs. The Gaussian filter is applicable to both optical images and LiDAR data. Two parameters, the window size and the standard deviation of the Gaus- 


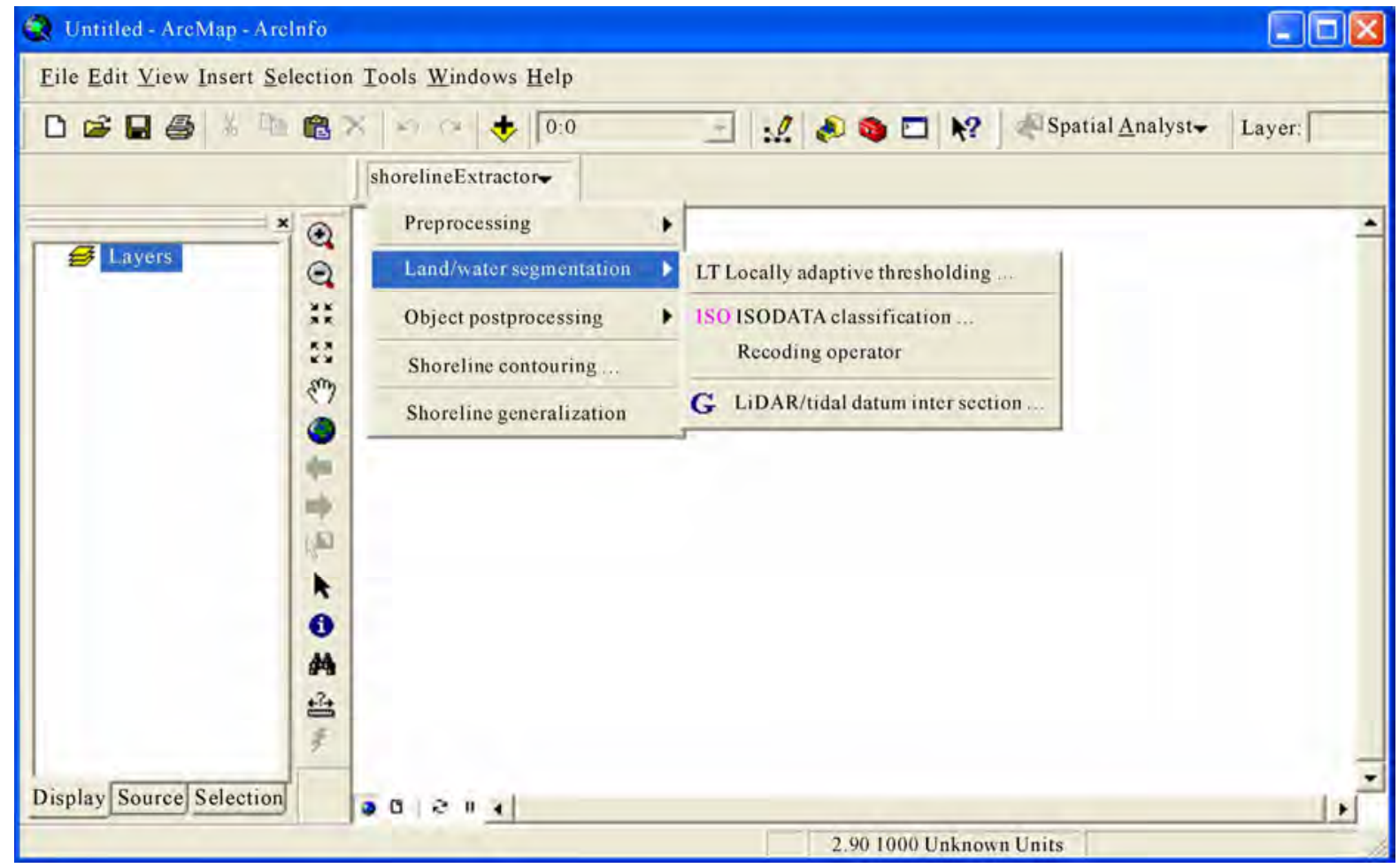

Figure 2. Graphical interface of the ArcGIS shoreline extraction extension module-shoeline extractor.

sian, need to be specified by the user. This parameter determines the degree of smoothing. A Gaussian filter with a large standard deviation requires a proportionally large convolution kernel to represent the weights precisely.

2) Lee Sigma Filter: This filter has been specifically designed to reduce speckle noises in radar images. Speckle is the grainy salt-and-pepper noise (exceptionally low or high pixel intensity values) in radar imagery due to random constructive and destructive interference of coherent radar signals from target scatters. Basically, radar speckle has the nature of a multiplicative noise. The Lee Sigma filter [34] assumes that a certain percentage of random samples within the range of $m \pm k \sigma$ are free from speckle contamination, where $m$ is the mean and $\sigma$ is the standard deviation (sigma). This filter replaces each pixel with the mean of all DN values in the moving kernel that fall within the designated standard deviation range $(m \pm$ $k \sigma$ ), in which the pixels beyond the standard deviation range are regarded as speckle-contaminated and hence not used to calculate the mean. The Lee Sigma filter is capable of reducing radar noise and speckle without degrading the sharpness of the shoreline edges. This filter is implemented by a VB.NET program calling the raster ArcObjects and invoking the $\mathrm{C}++$ program that implementing the algorithm. Two parameters, kernel window size (with a default value of 3) and sigma multiplier ( $k$ ) (with a default value of 2), need to be specified by the user. If the window size is larger or the sigma multiplier $(k)$ is smaller, the noise filtering effect is stronger.

3) Median filter: The median filter replaces each pixel in the image with the median of those values within the moving kernel. The median filter is applicable to optical images, radar images as well as LiDAR data. It is effective in removing white noise and salt-and-pepper radar speckles, while preserving sharp shoreline edges. It is implemented through a VB .NET program calling the ArcObject. Only one parameter, the kernel window size (with a default value of 3) needs to be specified by the user.

4) Anisotropic diffusion operator: The nonlinear anisotropic diffusion operator was originally proposed by Perona and Malik [35] for suppressing image noise and unwanted edges. It computes the diffused pixel value within a $3 \times 3$ kernel in an iterative fashion $[27,35]$. The directional diffusion coefficients are determined by the gradients between the central processing pixel and its four immediate horizontal and vertical neighbor pixels. By specifying a gradient threshold value $(K)$, the diffusion operator retains and enhances strong edges with a gradient greater than $K$ while suppressing and smoothing noise and weak edges with a gradient smaller than $K$. We implement the anisotropic diffusion algorithm using the $\mathrm{C}++$ language as a DLL. The software routine is developed through a VB.NET program calling the DLL file and the relevant ArcObjects. Its dialogue menu is shown 
in Figure 3(a). The two parameters, the gradient threshold $(K)$ (with a default value of 8 ) and the iteration number (with a default value of 5), need to be specified by the user. The larger the gradient threshold value or the iteration number, the stronger the smoothing effect. The appropriate choice of the gradient threshold value can achieve the intended objective of enhancing the major edges along the shoreline features while suppressing noise, interior variations, and unimportant weak edges inside land or water objects [27].

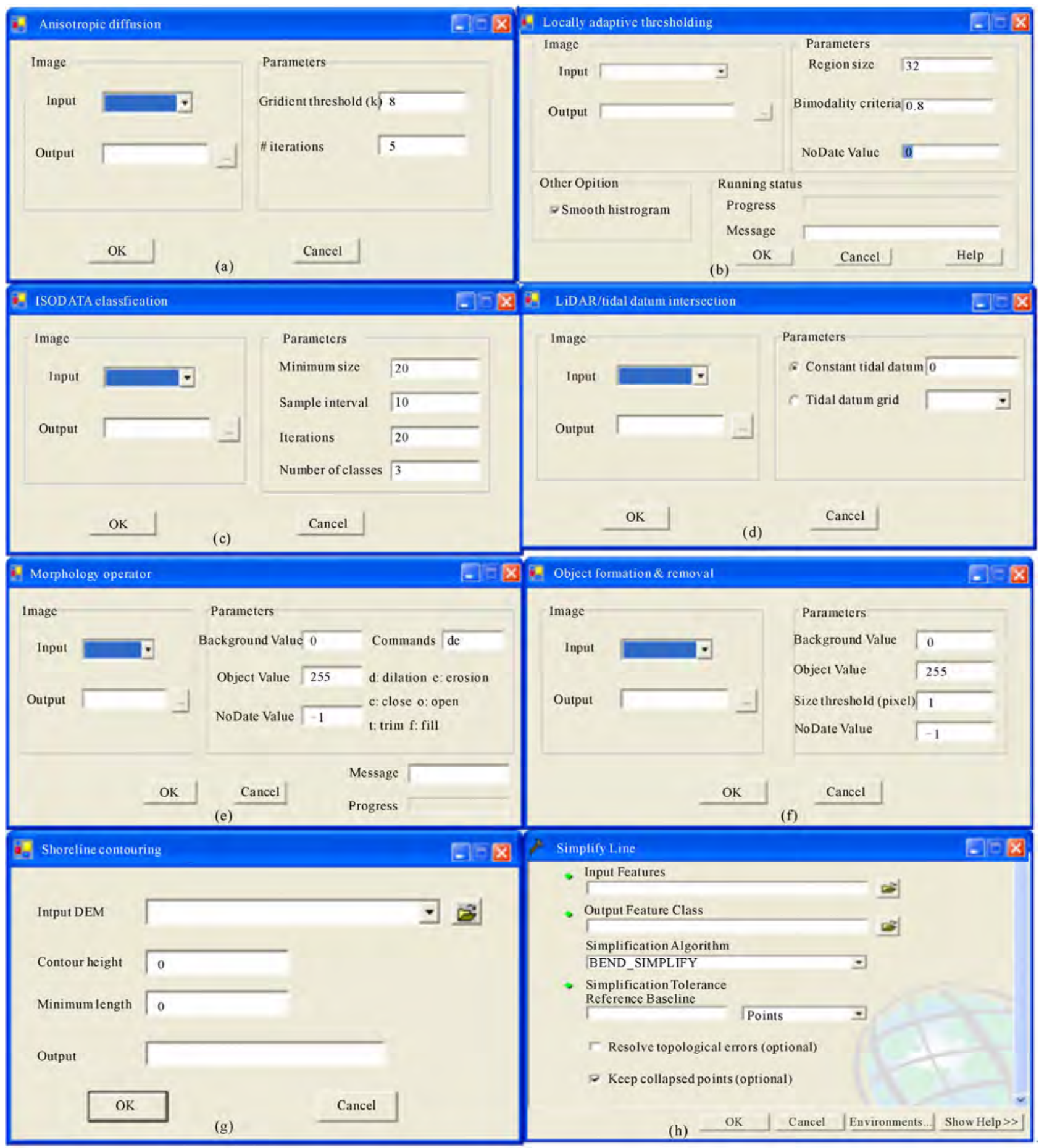

Figure 3. Graphical interfaces for selected shoreline extraction routines. (a) anisotropic diffusion routine; (b) locally adaptive thresholding routine; (c) ISODATA classification routine; (d) LiDAR/tidal datum intersection; (e) morphology operator routine; (f) object formation and removal routine; (g) shoreline contouring routine; and (h) shoreline generalization. 


\subsection{Algorithms and Routines for Segmenting and Classifying Land and Water Pixels}

Forming homogenous land and water objects for shoreline extraction requires an accurate and reliable differentiation of land pixels from water pixels. The border pixels between segmented land/water objects can be then delineated as the shorelines. The separation of an image or a LiDAR DEM into constituent land and water parts is the most important step in the object-based approach. Three algorithms and software routines are implemented for this purpose. Those include the locally adaptive thresholding, ISODATA classification followed by a recoding operation, and intersection of LiDAR grid and the selected tidal datum. The locally adaptive thresholding routine is designed to segment a panchromatic or single band image into land and water pixels. The ISODATA classification routine can classify a color or multi-spectral imagery into a number of land cover clusters, which can be subsequently combined into two categories (land and water pixels) through the recoding routine. The LiDAR grid and tidal datum intersection routine compares LiDAR elevation values with the corresponding tidal datum values and classify LiDAR elevation grid cells into land and water pixels.

1) Locally adaptive thresholding: it is the key algorithm component for segmenting a panchromatic or single band image into land and water pixels. The underlying concept is that the histogram of a small image region that contains a shoreline can be characterized by a mixture of double Gaussian distributions. First, the entire image is subdivided into a set of small, overlapping, square regions. For each small region, we examine the bi-modality and analytically determine a local threshold value to separate the land pixels from the water pixels. If the small image region consists solely of land or water pixels, the probability distribution of the intensity values will be uni-modal. If the image region consists of both land and water pixels, namely, the region contains shoreline features, the intensity values of land pixels and water pixels will be grouped into two dominant modes (bi-modality) with distinct mean values. The overall histogram for this region would exhibit two peaks and a valley. The lowest histogram valley point is used as a threshold value to reliably classify the pixels into land and water pixels.

To computationally determine the valley point, the bimodal histogram is modeled with a mixture of two Gaussian (normal) distribution functions, in which there are five unknown parameters to be determined [28,36]. The Levenberg-Marquardt algorithm is used to iteratively compute the optimal estimates for the five parameters based on the observed histogram [28], and op- timal threshold value can be computed analytically.

For all the small image regions whose histograms have appreciable bi-modality [28,36], optimal local thresholds can be computed using the process described above. As a result of processing all the image regions, a set of irregularly distributed threshold values are obtained. Next, an Inverse Distance Weighted (IDW) interpolation method [28] is employed to interpolate these irregularly distributed threshold values into a threshold grid. In other words, a threshold value is estimated for every pixel based on the threshold values of adjacent image regions using spatial interpolation. Subsequently, all the image pixels are segmented into land and water pixels by comparing their intensity values with corresponding local threshold values. If a pixel has an intensity value above its local threshold, it is flagged as a land pixel. Otherwise, it is designated as a water pixel. The locally adaptive thresholding algorithm can be summarized into the following computational steps:

1) Divide the input image into a set of small, overlapping, square image regions;

2) Construct an observed histogram for each image region, and optionally smooth the histogram using a onedimensional Gaussian filter;

3) Estimate initial values for the five parameters of the bimodal Gaussian curve and use the Levenberg-Marquardt algorithm to iteratively fit the bimodal Gaussian parameters for each region;

4) Test the resulting Gaussian curve for bi-modality;

5) Based on the fitted estimates for the five bimodal Gaussian parameters, calculate the optimal local threshold for any region whose histogram passes the bi-modality test;

6) Repeat steps (2)-(5) until all image regions are processed;

7) Interpolate local thresholds into a threshold grid with the IDW algorithm; and

8) Segment the entire image into land or water pixels by comparing the intensity values with their local threshold values.

If a single global threshold were used for the entire image, some shoreline edges would remain undetected due to the heterogeneity of the intensity contrast, causing discontinuous shoreline edges in low contrast areas and inconsistency of shoreline edge positions between high contrast and low contrast areas [28]. Since the locally adaptive thresholding algorithm sets the threshold value dynamically according to the local image statistical properties, a good separation between the land and water can be achieved.

We implement the locally adaptive thresholding algorithm using the $\mathrm{C}++$ language as a DLL. A VB.NET program is written to call this DLL and relevant Ar- 
cObjects for the development of a graphical interface. The dialogue menu is shown in Figure 3(b). Two critical parameters need to be specified by the user for this routine. One is the width of the square image regions used to subdivide the image. The width of the regions is specified in pixels with a default value of 32. The width should be small enough so that only one or two categories of pixels exist in the region. It also should be big enough to ensure reliable statistical analysis of the histogram. Normally, the larger the width, the more generalized the resulting land and water objects. The other parameter is the bi-modality criterion indicated by a minimum value of the valley/peak ratio (valley height/lower peak height). Optimal local threshold values would be analytically determined only for those image regions whose histograms have a valley/peak ratio larger than the minimum value. The default value for the bi-modality criterion is set as 0.8 . A lower criterion value will result in fewer but more reliable optimal thresholds to be computed. The smoothing of the histogram can speed up the convergence of the iterative computation of the Levenberg-Marquardt algorithm, if the image is noisy. Whether or not to smooth the observed histogram for each image region is controlled by a check button on the dialogue menu.

2) ISOADATA classification and recoding operator: The Iterative Self-Organizing Data Analysis Technique (ISODATA) [37] is a popular unsupervised classification method. With the ISODATA classification algorithm, a color or multi-spectral remote sensing image can be classified into a number of surface cover types, which can be further merged into two broad categories, land and water pixels, by using a recoding operator.

ISODATA classification performs an iterative, optimization clustering of a multi-spectral image. First, it arbitrarily assigns initial mean values for a specified number of clusters with equal interval that is defined by the mean and standard deviation of each band of the multi-spectral image. The region in the spectral space is defined using the mean and standard deviation of each band. In the first iteration, the multi-spectral values of each candidate pixel are compared to the mean values of different spectral bands of each cluster. The Euclidean distance between the multi-spectral values of each pixel and the mean values of each cluster is calculated. The candidate pixel is assigned to the cluster whose Euclidean distance is the shortest from the pixel. In the second iteration, new mean values are re-calculated for all the spectral bands of each cluster based on the pixels actually assigned to each cluster using the minimum Euclidean distance rule in the first iteration. The formed clusters are then diagnosed to decide whether or not they need to be merged or split. If a cluster contains less than the minimum percentage of pixels, it would be merged with the nearest cluster. If the Euclidean distance between the mean values of two clusters is below a threshold (default value of 3), these two clusters will be merged. If the standard deviation for a cluster exceeds a specified maximum standard deviation (typically between 4.5 and 7) or the number of pixels in a cluster is greater than the specified maximum number, the cluster will be split into two clusters. Mean values for the two new clusters are calculated as the mean of the original single cluster plus or minus the standard deviation. After the merging and splitting process, the mean values for new clusters are calculated. In the next iteration, every pixel in the image is once again assigned to a cluster using the minimum Euclidean distance rule. This iterative process is repeated until there is little change in class assignment between iterations or the maximum number of iterations is reached. At the final iteration, pixels are assigned to clusters using a maximum-likelihood decision rule based on the means and standard deviations of the spectral bands of each cluster.

We implement the ISODATA classification algorithm through a VB.NET program calling the available functions of ArcObjects. The dialogue menu for this routine is shown in Figure 3(c). Four parameters need to be set to run this routine: the number of output clusters, the number of iterations, minimum cluster size in pixel, and sample interval in pixel. The specified number of clusters (default value is 3 ) is the maximum number of clusters to be identified in the iterative clustering process. It is advised to enter a conservatively high number, analyze the resulting clusters, and to then re-run the function with a reduced number of classes. The number of the iterations (the default is 20) specifies the number of rounds to classify pixels and recalculate cluster mean values. The ISODATA algorithm terminates when this number is reached. This number should be large enough to ensure that after running the specified number of iterations, the migration of pixels from one cluster to another is minimal, and the clusters have become stable. Minimum cluster size (the default is 20) specifies the number of pixels to form a valid cluster, and clusters containing pixels fewer than this number will be merged with other clusters. This minimum cluster size should be about 10 times larger than the number of bands in the multi-spectral imagery in order to obtain reliable statistics. The sample interval (the default is 10) specifies the row and column interval between pixels that are actually sampled and used in the iterative computation of the mean and standard values of the spectral bands of the clusters. Larger sample intervals reduce the computation time but may introduce bias in the statistics.

The output of ISODATA routine is a classified image, 
and each pixel has an integer value to indicate the identification number of each cluster. A recoding routine is developed using a VB.NET program calling the relevant ArcObjects. The recoding routine can load and display the classified image from the ISODATA classification, and on the dialogue menu the user can specify which clusters should be categorized as land pixels and which as water pixels through visual inspection of the classified image. Therefore, the combination of ISODATA classification and the recoding operation creates a binary image consisting of land and water pixels.

3) LiDAR/tidal datum intersection: This routine aims to segment LiDAR elevation grid cells into land and water pixels by comparing the elevations with a tidal datum value. If a pixel has an elevation lower than the tidal datum value, it is coded as a water pixel. Otherwise, it is coded as a land pixel. The LiDAR elevation grid and the tidal datum grid must be referenced to a common datum [22]. This routine is implemented through a VB.NET program calling relevant ArcObjects. On the dialogue menu for this routine (Figure 3(d)), the input tidal datum surface can be specified as a constant value or loaded as a grid with varying values. For a small study area, the tidal datum can be treated as a constant valuable obtained from the nearest tidal gauge station. For a large study area, a tidal datum grid should be created by interpolating the tidal datum values measured by all tidal gauge stations in the study area.

\subsection{Algorithms and Routines for Post-Processing Land and Water Objects}

As described above, we can obtain a classified binary image consisting of land and water pixels by applying locally adaptive thresholding routine to a panchromatic or single band image, or by applying the ISODATA classification to a color or multi-spectral image followed by the recoding operation, or by intersecting a LiDAR DEM with a tidal datum surface. In the binary image, spatially connected land pixels form homogenous land objects, and spatially connected water pixels constitute homogeneous water objects. Misclassifications may occur, due to data noise, insufficient or inconsistent contrast between land and water, and the complexity of the scene. For instance, wet surfaces, cloud shadows, and building shadows are often misclassified as water objects, and whitewater, foam, ships, oil rigs, and clouds are often misclassified as land objects. The primary characteristic of these misclassified objects is that their areal size is significantly smaller than that of true land or water objects. To correct misclassifications and achieve a reliable shoreline, land and water objects need to be further processed before extracting the boundaries as shorelines.
Three software routines are devised to achieve this goal: morphology operator, object identification and spurious object removal, and object boundary tracing. The morphology operator $[38,39]$ aims to smooth the boundaries of land and water objects. The object identification and spurious object removal routine is intended to explicitly form image objects and then eliminate misclassified and unwanted objects. The object boundary tracing routine is used to delineate the boundaries between land and water objects as shoreline features and output them as ArcGIS vector data (ERSI Shape files or geodatabase feature class).

1) Morphology operator: Because of data noise and resolution limitation, land and water objects created in the segmentation and classification stage often exhibit noisy and jagged boundaries. Many tiny inlet- and peninsular-like features along the boundaries are spurious. The morphology operator is designed to smooth the boundaries and eliminate erroneous small-scale inletand peninsular-like features. The morphology operator is based on two fundamental operations: dilation and erosion [33,38,39]. Dilation adds pixels to the perimeter of each image object, generally increasing the size of objects, thus potentially filling small holes and broken areas, and connecting disjoint objects that are separated by spaces smaller than the size of the structuring element. Erosion etches pixels away from the perimeter of each image object and therefore shrinks the object. The operations of dilation and erosion can be combined into more complex sequences. The most useful combinations for morphological filtering are known as opening and closing. Opening consists of an erosion followed by a dilation and can be used to eliminate all pixels in regions that are too small to contain the structuring element. Closing consists of a dilation followed by erosion and can be used to fill in holes and close small gaps. We implement the morphology operator using the $\mathrm{C}++$ language as a DLL. A VB.NET program is developed to call this DLL and relevant ArcObjects. The dialogue menu for this routine is shown in Figure 3(e). Closing, opening, and any sequential combination of dilation and erosion operations can be specified on this menu. In addition, two simple morphology operations, fill and trim, are also added as alternative options on the menu. The fill operation fills single-pixel interior holes or single-pixel dents and cavities on the boundary. The trim operation cuts off single pixel protrusions and overhangs on the boundary.

2) Object formation and spurious object removal: The fundamental observation is that real land and water (ocean) masses usually form large, continuous image objects. Erroneous and misclassified image objects commonly have a significantly smaller size. Explicitly grouping connected land and water pixels into individual 
image objects and deriving their geometric properties renders the capability of discriminating erroneous and misclassified objects from true land and water objects based on the heuristic knowledge about the size and continuity of land and water bodies in the study area. A land object consists of a set of spatially connected land pixels. Two land pixels are defined to be connected if one is located in the immediate neighborhood of the other. A recursive expansion algorithm is used to identify and index image objects [28,33]. First, the image is scanned in a row-wise manner, and a seed is set at the first land pixel, which is treated as a single pixel land object. This single pixel object is expanded to include all land pixels located in the immediate neighborhood of the current land pixel. The expansion is continued recursively until all connected land pixels are included. This recursive expansion process may be repeated to identify other land objects. The land objects are indexed with a unique integer, starting with 1 . During the object formation and indexing process, the areal size and other geometric properties of each object are also calculated. Small non-water objects scattered in the water bodies often correspond to ships, oil rigs, ice bergs, clouds, whitewater, foam, or image noise. Since the boundaries of these small image objects are not true shorelines, they should be selected with an area threshold and eliminated by fusing them into the water bodies. Similarly, water objects can be explicitly identified and indexed, and small noisy water objects corresponding to wet surfaces, building and cloud shadows, and data noise can be dissolved into the land with another user specified areal threshold value.

We implement the object identification and spurious object removal routine using the $\mathrm{C}++$ language as a DLL. The software routine is developed through a VB.NET program invoking the DLL and calling ArcObjects. The dialogue menu for this routine is shown in Figure 3(f). The user needs to specify the object value and background value. The parameter of the areal size threshold value in pixel needs to be specified by the user to remove small noisy image objects. In practice, this routine is run in two passes. Assume that in a segmented image the land pixels are coded by the value of 255 and water pixels are coded by the value of 0 . In the first pass, by specifying the object value to be 255 and background value to be 0 , land objects are identified and spurious objects specified by the size threshold value are removed. In the second pass, by specifying the object value to be 0 and the background value to be 255, water objects are identified and small water objects specified by the size threshold value are eliminated. After two passes of selective removal of small, isolated, and noisy image objects, only large continuous land and (ocean) water objects are left, which define true shorelines. This routine can effec- tively eliminate unwanted, misclassified objects whose boundaries are not shorelines. This greatly reduces the editing cost for cleaning up the final shoreline product.

3) Boundary tracing and vectorization: with the object based approach, the shoreline is defined as the boundary between land and water objects. Each land pixel in an image object is scanned by a $3 \times 3$ neighborhood window to examine its four immediate neighbors (horizontal and vertical). If one or more neighbors of the land pixel belong to water (background) pixels, this land pixel will be flagged as a boundary pixel. In this way, all land pixels immediately adjacent to the water pixels are extracted as boundary pixels. Then, a recursive algorithm is used to trace boundary pixels into a set of vector lines. The output of this routine is an ArcGIS Shape file containing vector shorelines, which can be directly displayed, validated and edited in the ArcGIS environment.

\subsection{Algorithm and Routine for Contouring LiDAR Data for Shorelines}

Shorelines can be derived from LiDAR data alternatively by using a contouring method. First, the LiDAR data need to be adjusted with reference to the tidal datum surface. After subtracting the tidal datum from the LiDAR DEM, the resulting zero elevation cells can be contoured to represent the tidal-datum referenced shorelines. Although the contouring routine is available in most GIS software packages, it is designed for a general topographical mapping purpose. To use such a routine, the user has to specify a base contour line and contour interval, resulting in a series of contour lines with incremental elevation values. Since these contouring routines are not optimized for shoreline extraction, they often produce many short, broken, and noisy shoreline segments when applied to LiDAR data [21,30,31]. Therefore, a great deal of manual editing or re-digitizing work was involved in creating the final clean shoreline representation [21,30,31].

To overcome the difficulties, we develop a dedicated contouring routine for shoreline extraction. This routine only traces connected cells with a specified elevation value (zero in most cases). A constraint on the length of the traced shoreline segments is also imposed for the contouring process. Noisy and broken dangle lines will be dropped automatically as long as their length is shorter than a user specified threshold value. We develop this software routine using the VB .NET to call ArcObjects. The dialogue menu for this routine is shown in Figure 3(g). Two parameters can be specified by the user. One is the elevation to be contoured, and its default value is zero. The other one is the minimum length of shorelines to be kept. The output of this routine is a 
shape file of shorelines, which can be display and edited further in ArcGIS.

\subsection{Algorithms and Routines for Shoreline Generalization}

Two algorithm options are included in the shoreline smoothing and generalization routine. The first is the Douglas-Peucker algorithm for line simplification. It keeps the critical points that depict the essential shape of the shoreline and removes redundant details such as extraneous bends, fluctuations, small intrusions and extrusions. The algorithm connects the end-nodes of a curve segment with a trend line. The distance of each vertex to the trend line is measured perpendicularly. Vertices with a distance to the line less than the specified tolerance are eliminated. The algorithm is efficient for data compression, but the resultant shorelines may contain unpleasant sharp angles and spikes which reduce the cartographic quality. The second algorithm simplifies the bends on the shorelines. It analyzes the shape of the shorelines and identifies the high-curvature bends. Insignificant extraneous bends are then removed, and too narrow bends are slightly widened to satisfy the tolerance. Compared with the Douglas-Peucker algorithm, the bend simplification algorithm tends to produce smoother and hence cartographically more appealing shorelines. We implement this software routine through a VB.NET program calling the ArcObjects. Through the dialogue menu, the user can make a selection between the Douglas-Peucker algorithm and the bend simplification algorithm. The only parameter that the user needs to specify is the weeding tolerance value, which determines the degree of generalization.

\section{Shoreline Extraction Scenarios}

\subsection{Data Sources and Application Requirements}

From the data processing perspective, the possible input remote sensing data for shoreline extraction can be grouped into three categories: single band imagery, multi-band imagery, and LiDAR DEMs. The single band imagery can be panchromatic image, near infrared image, and Synthetic Aperture Radar image acquired by satellite or airborne sensors. Multi-band imagery can be natural color image, false color near infrared image, multi-spectral image, multi-polarimetric SAR image from satellite or airborne sensors.

In practice, the choice of data sources for shoreline mapping at a specific site is often dictated by the availability and cost of data. The land cover types in the coast zone and the requirements for spatial coverage and reso- lution of shoreline also influence the data selection. Although relatively small coastal areas are involved, most of coastal engineering applications require highly detailed and precise shoreline information, which can be only satisfied by aerial photographs in the past. The successful launches of IKONOS satellite in September 1999, QuickBird satellite in October 2001, WorldView-1 satellite in September 2007, and GeoEye-1 Satellite in September 2008 have produced 1-meter or sub-meter satellite imagery, which can be used for high resolution coastal mapping applications. For coastal resource inventory and management, flood and hazard zone delineation, and other environmental applications, satellite images at fine or moderate spatial resolution, such as Landsat, SPOT, ASTER, and Radarsat SAR images, can be a good choice due to their extensive ground coverage and the repetitive acquisition. Coastal erosion and historical shoreline change studies require multi-temporal shoreline information. Historical data are limited or nonexistent at the great majority of coastal sites. Satellite image data did not exist until 1970s, while aerial photography began to be available for many areas of the coast in the 1940s [1,40], which is the most common data source for determining past shoreline positions. For coasts with sandy beaches or exposed rocks, all types of image data are applicable for discerning and mapping shoreline features. For shallow coastal waters with high concentration of suspended sediments or underwater features, shorelines can be mapped more easily and accurately from near infrared images and radar images than from panchromatic and natural color images, because the land-water contrast is much stronger on the infrared and radar images. For the low-lying coasts with wetlands, mangroves and other types of vegetation, the use of color near infrared aerial photographs and multispectral satellite images would make the separation of water from land easier and more accurate, with a result of better determination of shoreline position.

Airborne LiDAR promises an accurate and cost-effective approach to coast and shoreline mapping [20,21]. Airborne LiDAR data have been increasingly available for the coastal applications since 1990s. The NOAA Coastal Services Center (CSC) has collected topographical LiDAR data at $1 \mathrm{~m}-2 \mathrm{~m}$ resolution along the United States coasts through a partnership program with the USGS Center for Coastal and Regional Marine Studies and the NASA Goddard Space Flight Center (http://www.csc.noaa.gov/crs/tcm/missions.html). For a number of US coastal states, LiDAR data have been acquired for multiple time periods. The shoreline position is constantly changing, because of cross-shore and alongshore sediment movement in the littoral zone and especially because of the dynamic nature of sea water 
levels (e.g., waves, tides, river discharges, storm surge, etc.) [30]. Since aerial photographs and satellite images are rarely taken at a water level of desired tidal datum elevation, it is often difficult to obtain tidal-datum referenced shorelines from image data. If LiDAR data are collected when the water level of sea surface is close to a minimum elevation (e.g. neap tide) with low wave energy, a set of shorelines with reference to various tidal datums, namely Mean High Water (MHW), Mean Higher High Water (MHHW), Mean Sea Level (MSL), Mean Low Water (MLW), or Mean Lower Low Water (MLLW), can be derived. Airborne LiDAR data not merely provide an efficient approach to the shoreline mapping and shoreline change detection [21,22,29], but also allow for the detailed calculation of volumetric changes for coastal erosion analysis [41-44]. High-resolution airborne LiDAR data should be used to replace or complement image data for shoreline mapping, where and when they are available.

\subsection{Selection of Software Routines and Parameter Setting}

The data flow diagram in Figure 4 illustrates how the software routines could be utilized and combined for processing different types of input remote sensing data sources for shoreline extraction. The selection and com- bination of software routines largely depend on the type of input data. In the data preprocessing stage, Gaussian filter can be applied to optical images and LiDAR data but are not recommended for radar images. Median filter is applicable to optical and radar images as well as $\mathrm{Li}$ DAR data. Lee Sigma filter is specially designed for removing speckle noise of radar images. Anisotropic diffusion operator can be applied to image data but not $\mathrm{Li}^{-}$ DAR data. In the land-water segmentation and classification stage, the locally adaptive thresholding routine is the choice for panchromatic aerial photographs, panchromatic or single band optical satellite images, and single band radar/SAR images. If the input data are natural color aerial photographs, color infrared aerial photographs, multispectral satellite images, multi-channel or multi-polarimetric SAR images, the ISODATA classification routine should be chosen to classify images into a number of land cover clusters, and then the recoding routine should be used to combine the land cover clusters into land and water pixels. When the input data are $\mathrm{Li}-$ DAR elevation grid, the LiDAR/tidal datum intersection routine can be used to separate LiDAR elevation grid cells into land and water pixels, or the contouring routine can be directly applied to extracting the shoreline.

Setting up the parameters for software routines largely relies on the noise level of input data, the scene complexity, and the desired generalization level and scale of

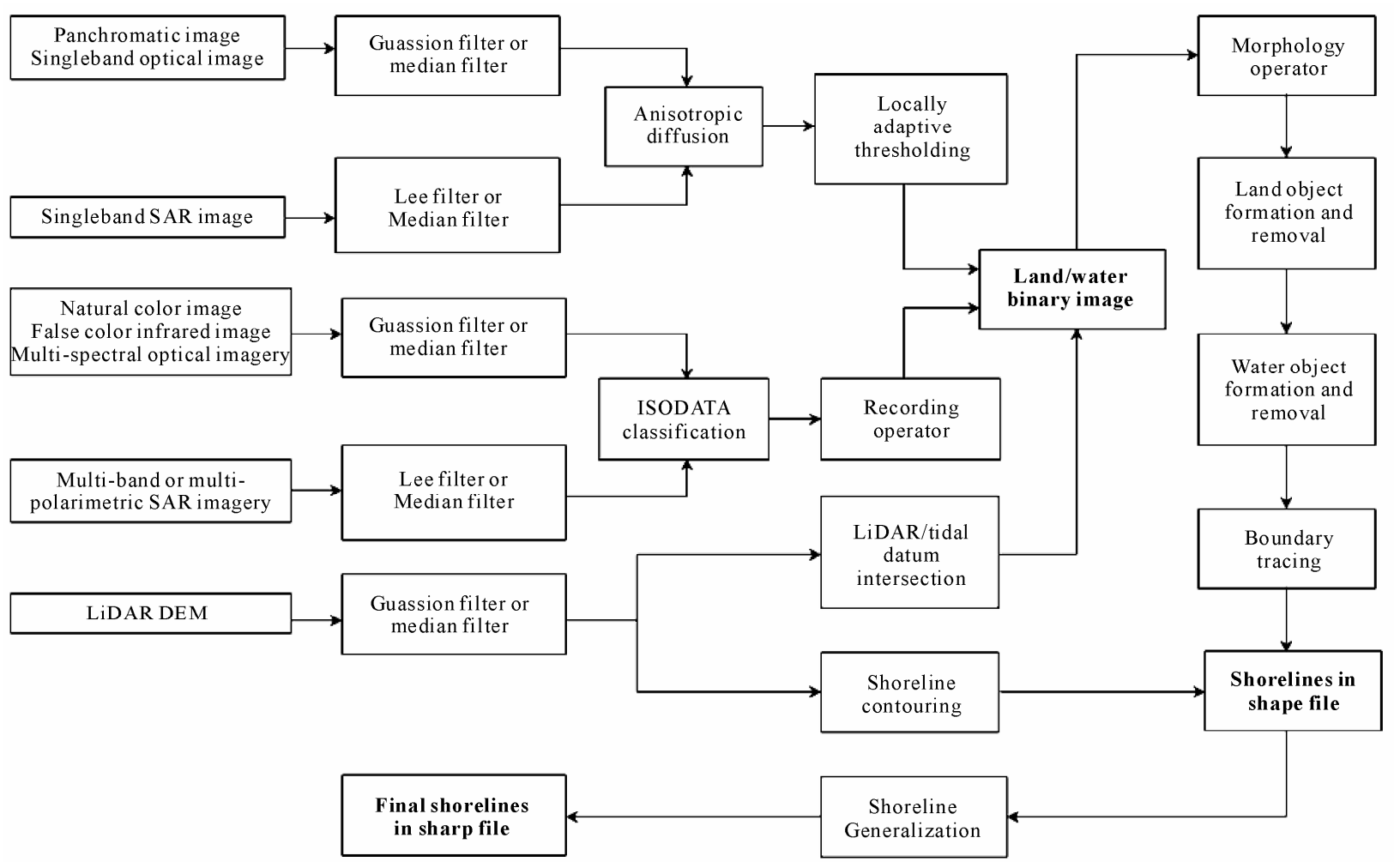

Figure 4. Data flow chart for processing different types of remote sensing image data and LiDAR data. 
the shoreline products. For the very noisy input data, filters with a large window size should be applied to enhance the noise smoothing effect. A filter can also be applied to the noisy input data for multiple rounds to achieve the desired smoothing effect. For a simple coastal scene containing only a few types of land cover, the width of the image region for histogram analysis in the locally adaptive thresholding routine should be set to a relatively large number, while the number of the output clusters in the ISODATA classification routine can be set to a relatively small number. In contrast, if the coastal scene is complex and contains many different land cover types, it is advisable to set the width of the image region in the locally adaptive thresholding routine to a relatively small number, and to set the number of the output clusters in the ISODATA classification routine to a conservatively high value.

The detail level of extracted shorelines depends largely on the spatial resolution of the original image or LiDAR data. Some applications may not need too much detail. In this case, the derived vector shorelines can be simplified and generalized to reduce data volume, to eliminate redundant and unnecessary details, and to improve the visual smoothness for cartographic representation. To increase the level of shoreline generalization, we can increase the size threshold in the spurious object removal routine, apply Douglas-Peucker routine or the bend simplification routine. Douglas-Peucker routine is efficient for data compression, but the resultant shorelines may contain unpleasant sharp angles and spikes. The bend simplification routine tends to produce smoother and hence cartographically more appealing shorelines.

In the following section, we will use Radarsat SAR image data, multi-spectral QuickBird data, and airborne LiDAR data to demonstrate how to use the shoreline extraction software routines and how to set up appropriate parameters for these routines.

\subsection{Application Examples}

\subsubsection{Shorelines from Single-Band Imagery}

A Radarsat SAR image over Galveston Bay, Texas is used to show how the software routines in the extension module can be used to derive shorelines from a single-band remote sensing image. The radar image was acquired on August 31, 2008 by a C-band SAR sensor on board Radarsat-2 satellite with an ultra-fine beam. The radar image has a spatial resolution of $3 \mathrm{~m}$. The SAR image was rigorously orthorectified and projected into the UTM (zone 15N) coordinate system with reference to WGS84 ellipsoid. The radar image shows sufficient contrast between land features and ocean water (Figure 5).
The data processing steps and software routines are as follows: smooth the Radarsat SAR image with the Lee Sigma filter routine (kernel window size $=5$, and sigma multiplier $k=2$ ); apply the anisotropic diffusion operator to enhance the shoreline edges and suppress the internal intensity variations inside the land and water bodies (the iteration number $=5$, and the gradient threshold value $=$ 20); apply the locally adaptive thresholding routine to segment the image into land and water pixels (with the width of the image regions $=128$ pixels); apply the morphology operator with the option of the close, trim, and fill operation) to the segmented image; apply object formation and spurious object removal routine to identify water objects and eliminate water objects with an areal size less than 5,000 pixels; apply the object formation and spurious object removal routine for the second pass to identify land objects and eliminate land objects smaller than 5,000 pixels; apply the object boundary tracing routine to create the shorelines as an ArcGIS Shape file, and apply shoreline generalization routine with the band simplification option (with weed tolerance value of $10 \mathrm{~m}$ ). Figure 5 shows the processing results for an enlarged portion of the image. Visual examination shows that the detailed shoreline features such as small inlets, islands, lakes, docks, piers and other subtle features have been faithfully and accurately marked out. To evaluate the positional accuracy, we compared the algorithm-derived shorelines with those visually interpreted by a careful human operator for three selected shoreline segments, each with a length of $150 \mathrm{~m}$. For these three shoreline segments, the algorithm derived shorelines closely matches those obtained from human visual interpretation, the Root Mean Squares Error (RMSE) for the derived shoreline position is 1.37 pixels, $4.1 \mathrm{~m}$ in this case. It should be noted that manually traced shorelines are generally robust and reliable without gross error, but less precise than numerically derived results.

\subsubsection{Shorelines from Multi-Band Imagery}

A multi-spectral QuickBird imagery over the North Sound, Antigua is used to demonstrate how the software routines can be applied to a multi-band image for shoreline extraction. The multi-spectral QuickBird image contains four bands (blue, green, red, and near-infrared) with a spatial resolution of $2.44 \mathrm{~m}$. The image was acquired on January 15, 2005. The full image scene covers about $16.5 \mathrm{~km} \times 16.5 \mathrm{~km}$ ground area. We performed the orthorectification of the basic imagery product using the supplied Rational Polynomial Coefficients (RPCs) and the SRTM DEM data. The orthorectified image has a horizontal position accuracy of about $15 \mathrm{~m}$.

The following data processing steps and software routines are used to process the multi-spectral QuickBird 


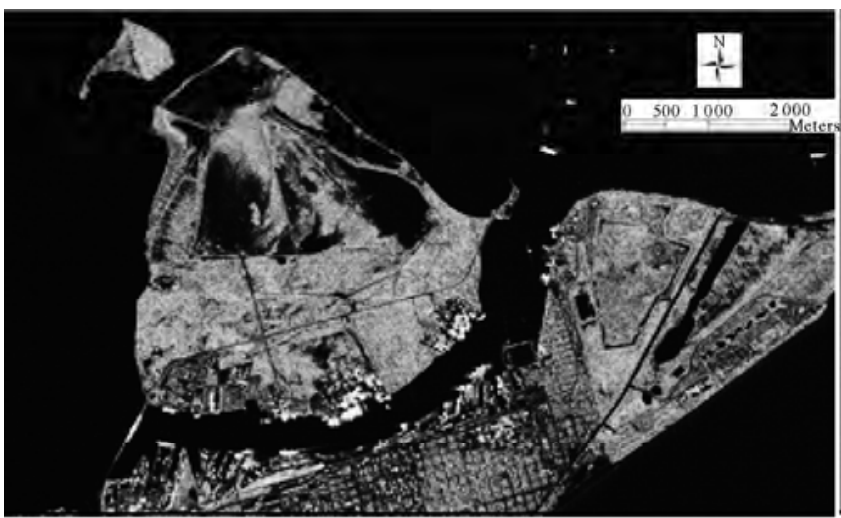

(a)

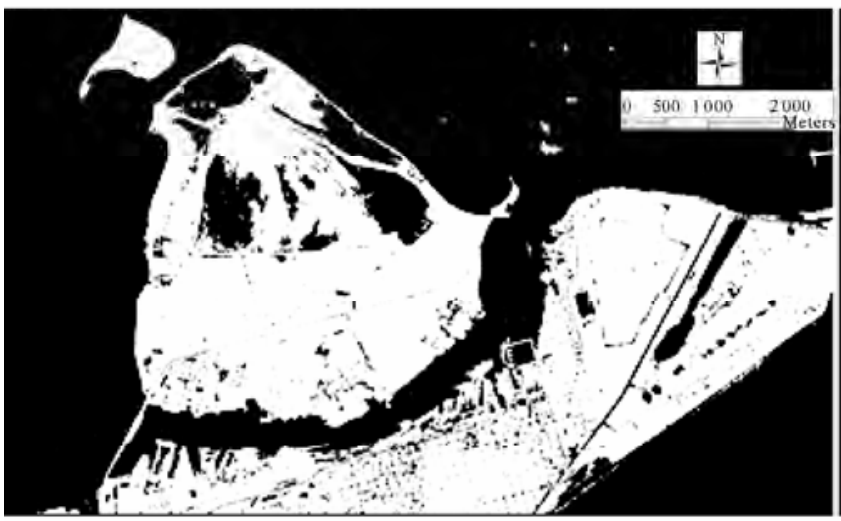

(c)

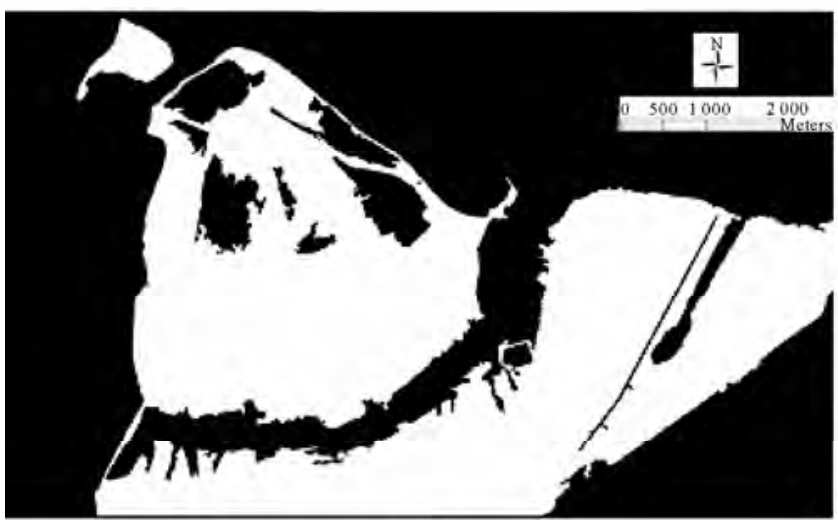

(e)

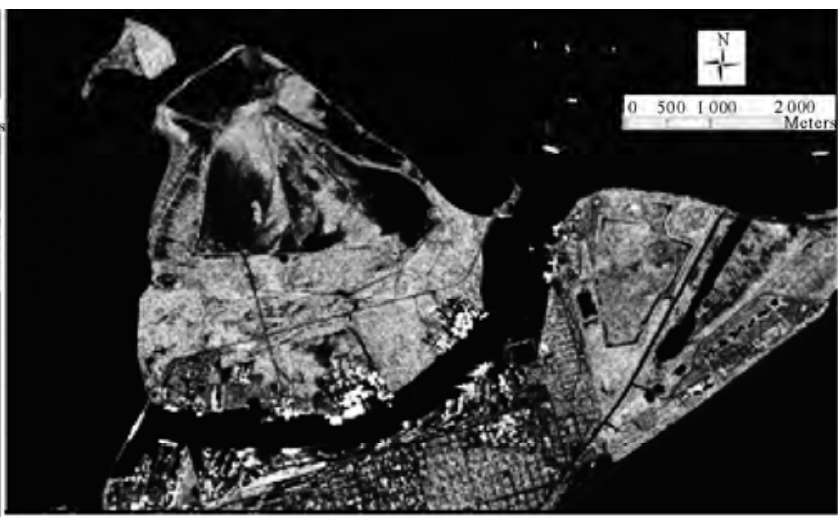

(b)

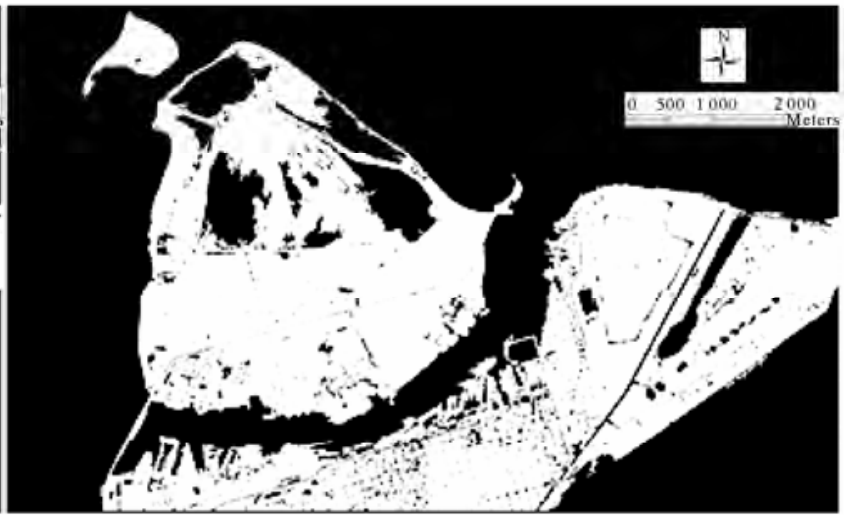

(d)

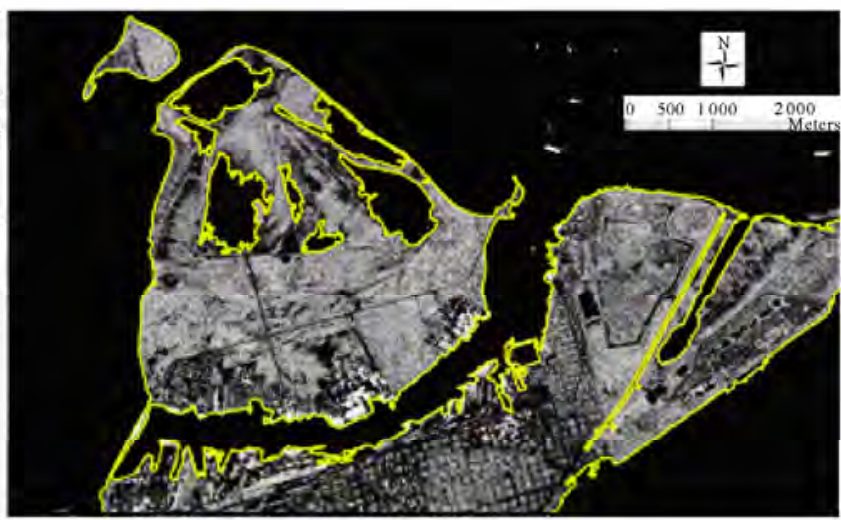

(f)

Figure 5. Shoreline extraction from Radarsat SAR image. (a) Original SAR image; (b) After applying Lee Sigma filter and anisotropic diffusion operation; (c) thresholding result; (d) After removal of small and noisy water objects with a threshold value of 5000 pixels; (e) After removal of small and noisy land objects with a threshold value of 5000 pixels; (f) Derived shorelines.

image: smooth the image with the Gaussian filter routine (the window size $=5$, the standard deviation $=1$ ); classify the multi-spectral image into 12 different types of clusters using the ISODATA classification routine (cluster number $=12$, iteration number $=30$, minimum size of cluster $=2000$ pixels, sample interval $=10$ ); combine different land cover clusters into land and water pixels using the recoding operator; apply the morphology operator with the option of the close, trim, and fill operation to the segmented image; apply object formation and removal routine to identify land objects and eliminate land objects smaller than 100 pixels; apply object formation and removal routine for the second pass to identify water objects and eliminate the water objects smaller 
than 2000 pixels; apply object boundary tracing routine to create the shorelines as an ArcGIS shape file, and apply the shoreline generalization routine with the band simplification option (with a weed tolerance value of 3 $\mathrm{m})$. Figure 6 shows the processing results for an enlarged portion of the image. We compared the algorithm-derived shorelines with those visually interpreted by a human operator for three selected shoreline segments, each with a length of $100 \mathrm{~m}$. The average accuracy (RMSE) of the derived shoreline position is 1.21 pixels, within $2.95 \mathrm{~m}$ in this case.

\subsubsection{Shorelines from Airborne LiDAR Data}

An airborne LiDAR DEM data set over the coastal zone of the Galveston Bay, Texas is used to illustrate the LiDAR data processing procedure for shoreline extraction with our software routines. The LiDAR data were acquired on October 16, 1999 by NASA's Airborne Topographic Mapper (ATM) laser instrument. Raw measurements of the LiDAR points used in this research have a horizontal accuracy of $0.8 \mathrm{~m}$ (RMSE) and a vertical accuracy of $0.15 \mathrm{~m}$ over the bare beach, and the spacing between original LiDAR sample points is between 1 and $2 \mathrm{~m}$ on the ground. The surveyed swath covers the beaches, foredunes, and a few rows of houses landward. The LiDAR DEM is projected to the UTM (zone 15N) coordinate system, horizontally referenced to the WGS84 ellipsoid. The elevation values of LiDAR DEM are adjusted to be referenced to the orthometric datum-the North American Vertical Datum of 1988 (NAVD88). Based on precise measurements of the horizontal and vertical positions of the benchmarks near tide gauge station-Galveston Pier 21, the tidal datum values (relative to NAVD88) for this area are determined as follows: 0.36 $\mathrm{m}$ for MHW, $0.387 \mathrm{~m}$ for Mean MHHW, $0.21 \mathrm{~m}$ for MSL, $0.048 \mathrm{~m}$ for MLW and $-0.043 \mathrm{~m}$ for MLLW. For this small study area, the tidal datum surface is assumed to be a level plane with a constant elevation.

First, the object-based approach is applied to the processing of the LiDAR DEM. The data processing chain is: apply a $3 \times 3$ median filter to reduce data noise; apply the LiDAR/tidal datum intersection routine to segment the LiDAR DEM into land and water pixels with the MHW tidal datum (0.36 m); apply the morphology operator with the option of the close, trim, and fill operation to the segmented image; apply the object formation and removal routine to identify land objects and eliminate the land objects smaller than 500 pixels; apply the object formation and removal routine for the second pass to identify water objects and eliminate the water objects smaller than 3000 pixels; apply the object boundary tracing routine to create the shorelines as an ArcGIS shape file, and apply the shoreline generalization routine with the band simplification option (with weed tolerance value of $3 \mathrm{~m}$ ). Figure 7 shows the processing results for the MHW datum referenced shorelines.

The second approach is contouring-based. The data processing sequence consists of the following steps: apply a $3 \times 3$ median filter to remove data noise; apply the contouring routine to trace the elevation values of the MHW datum (0.36 m) with a length threshold (1000 m) and save it as a ArcGIS shape file; and apply the shoreline generalization routine with the band simplification option (with weed tolerance value of $3 \mathrm{~m}$ ). Figure 8 shows the processing results with the contouring-based approach. Clearly, directly contouring without a length threshold creates many noisy, short line segments (Figure 8(b)) that are not true shorelines. With an appropriate length threshold $(1,000 \mathrm{~m})$, the noisy and erroneous shorelines can be avoided (Figure 8(d)). We repeat the above the processing steps with other two different tidal datums and produced the shoreline indicators for the MHHW $(0.388 \mathrm{~m})$ and MSL $(0.21 \mathrm{~m})$ datums as well (Figure 8(f)). For this LiDAR data set, the MLW (0.048 $\mathrm{m})$ and MLLW $(-0.043 \mathrm{~m})$ shorelines cannot be determined because the water level $(0.134 \mathrm{~m})$ on the LiDAR data acquisition day was above the MLW and MLLW datums.

It should be pointed out that the use of a constant tidal datum value for a large region could lead to the error in shoreline position determination. In the case of a large coastal region, a two-dimensional tidal datum surface should be computed by interpolating the tidal datum observations of available tidal gauge stations in the study area or modeled by a numerical hydrodynamic model [45]. Our accuracy analysis for the Upper Texas Gulf Coast suggests that the horizontal position of the shorelines derived from $1 \mathrm{~m}$ resolution LiDAR DEMs is accurate within $4.5 \mathrm{~m}$ at the $95 \%$ confidence level [22].

\section{Conclusions}

Coastal scientists have long recognized the importance of a rapid, accurate method for providing frequent and timely shoreline measurements, e.g. [46,47]. This paper identified the key algorithm components for automated shoreline extraction and implemented them as a series of software routines within an ArcGIS extension module "ShorelineExtractor". The combination of these software routines provides a powerful and flexible software tool, capable of processing various types of remote sensing imagery and LiDAR elevation data for shoreline extraction. Compared with conventional manual delineation methods, our automated shoreline extraction algorithms and software tools enjoy the advantages in efficiency, robustness, repeatability and objectivity. To our 


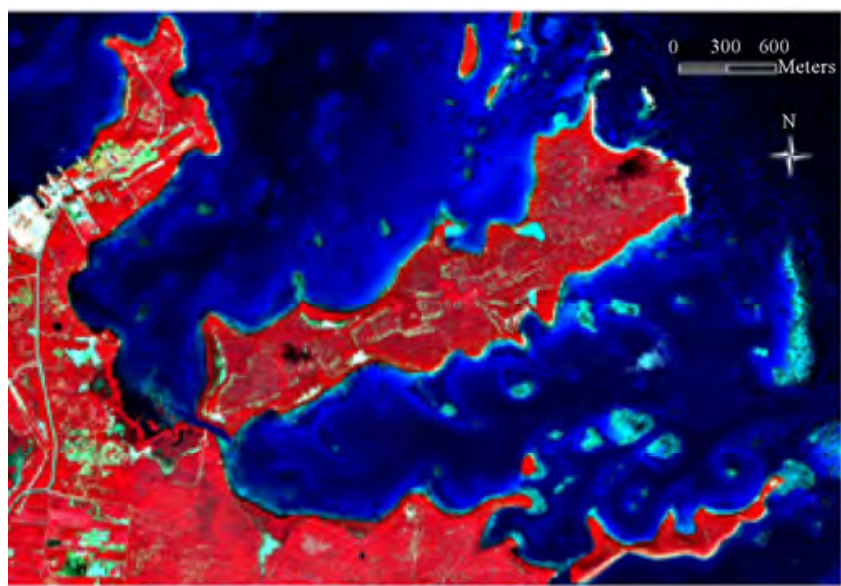

(a)

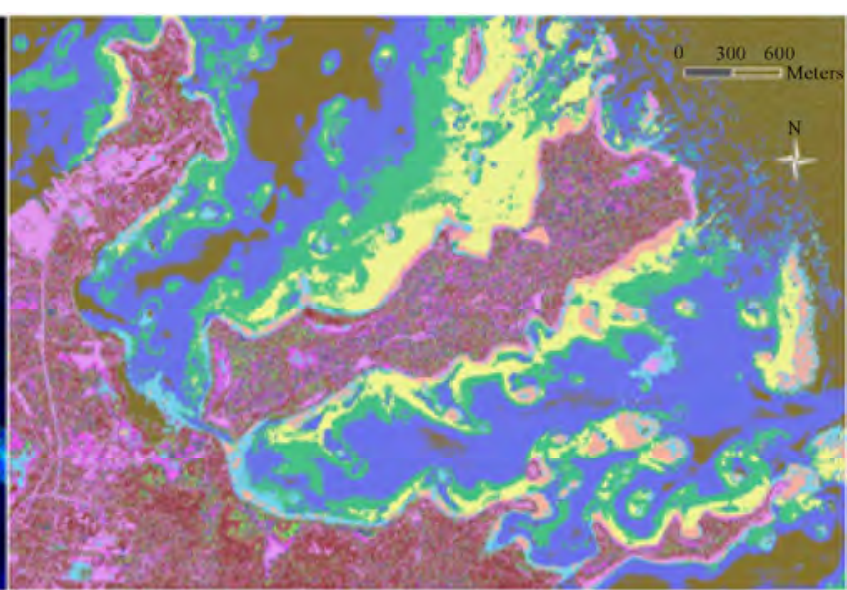

(b)

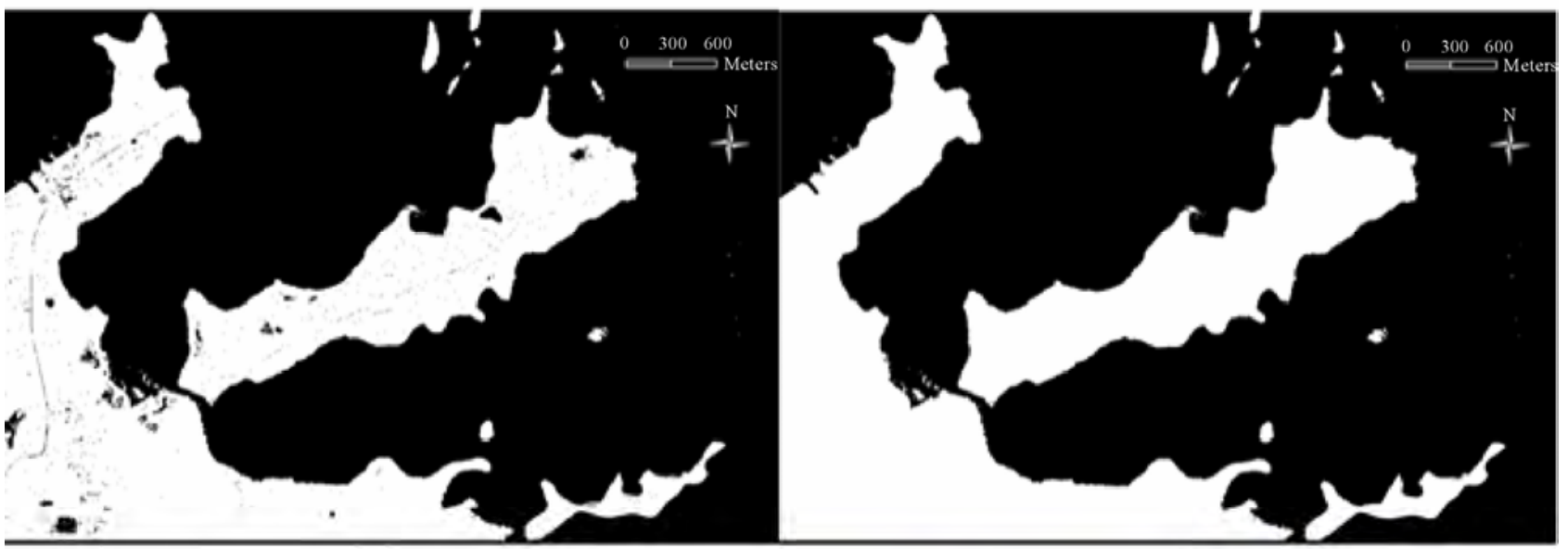

(c)

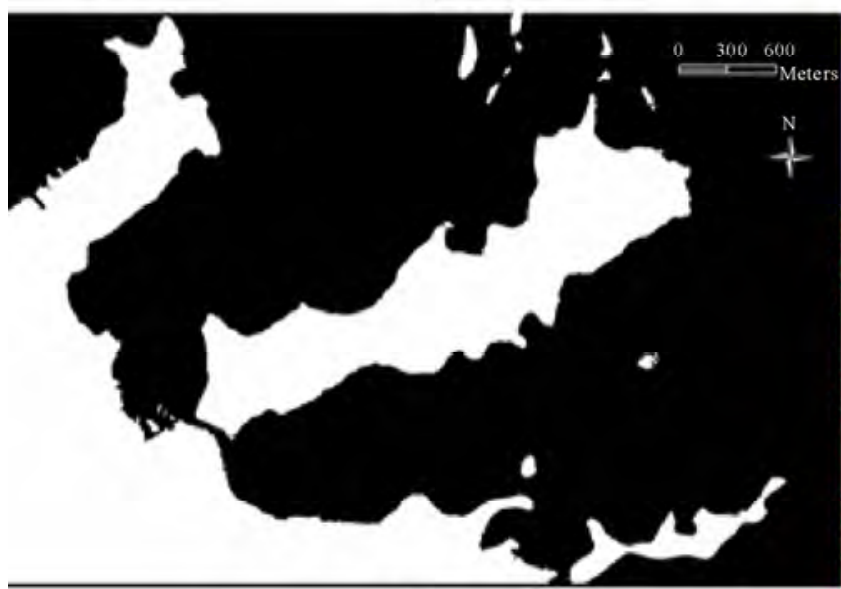

(e) (d)

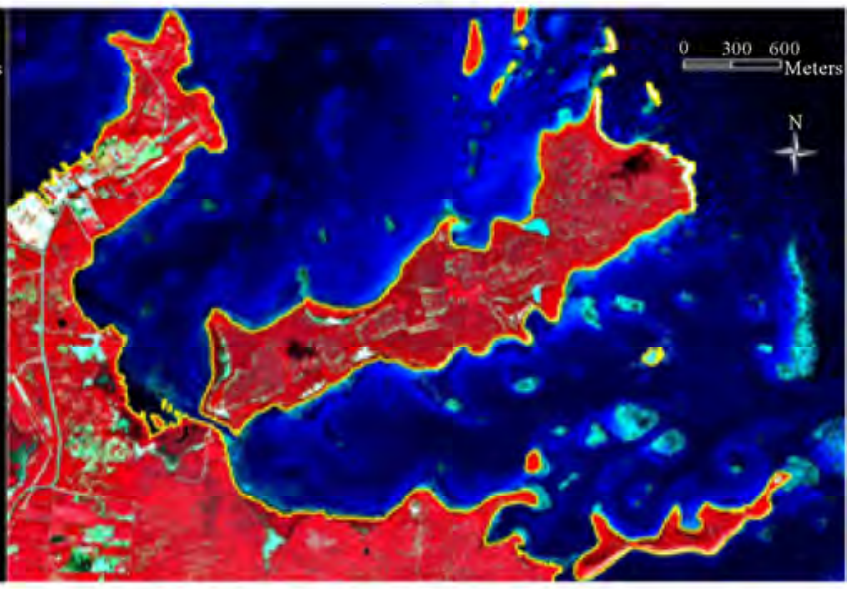

(f)

Figure 6. Shoreline extraction from multi-spectral QuickBird imagery. (a) Multi-spectral QuickBird image; (b) classification result (12 classes); (c) Recoding result; (d) After removal of small and noisy land objects with a threshold value of 100 pixels; (e) After removal of small and noisy water objects with a threshold value of 2000 pixels; (f) Derived shorelines.

best knowledge, our product is the first dedicated, comprehensive software package for extracting shorelines from remote sensing data.
We have presented insights and guidelines for selecting and combining different algorithm elements and specifying appropriate parameters for different software 


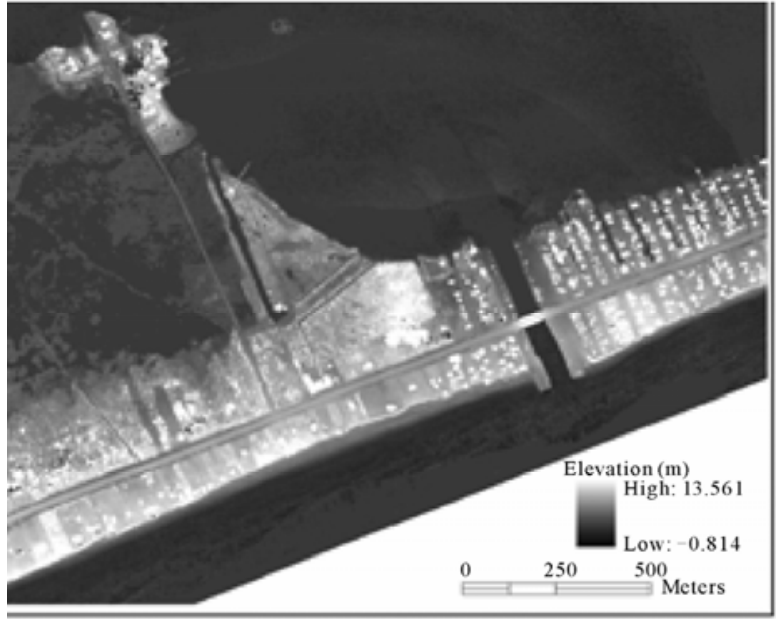

(a)

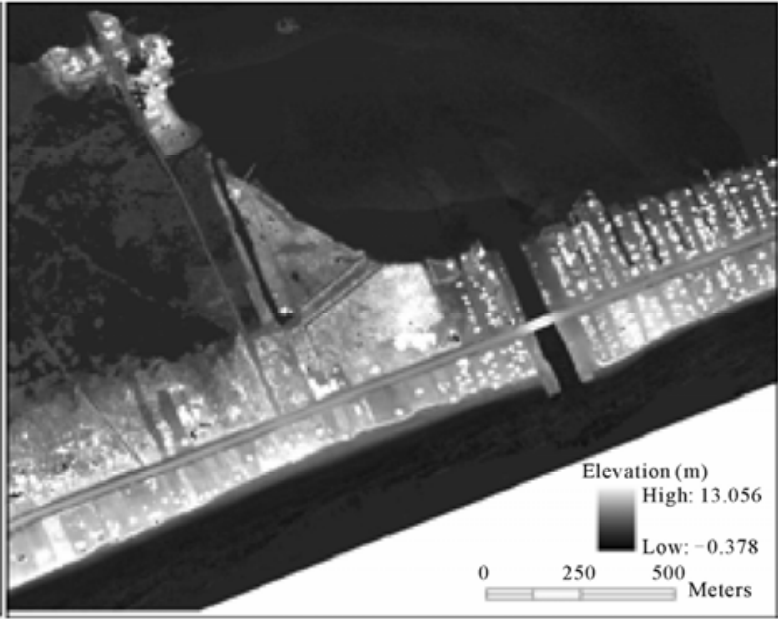

(b)

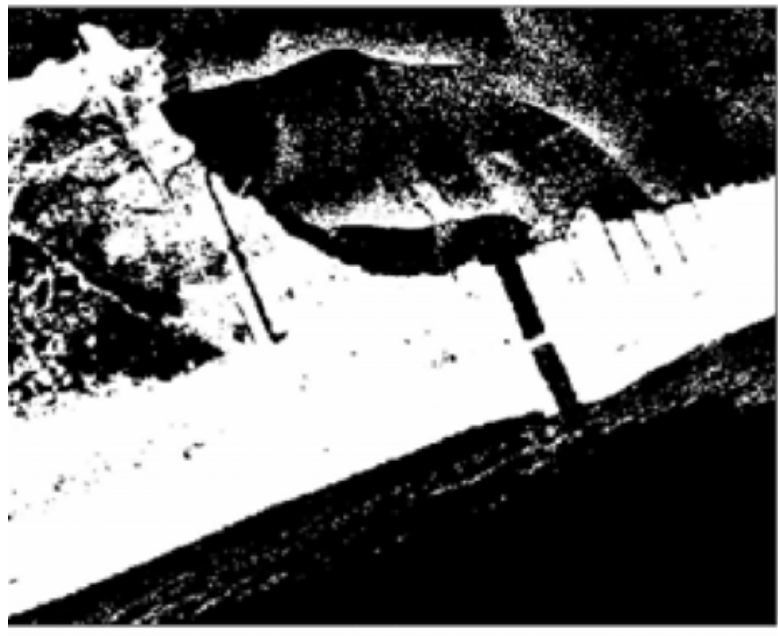

(c)

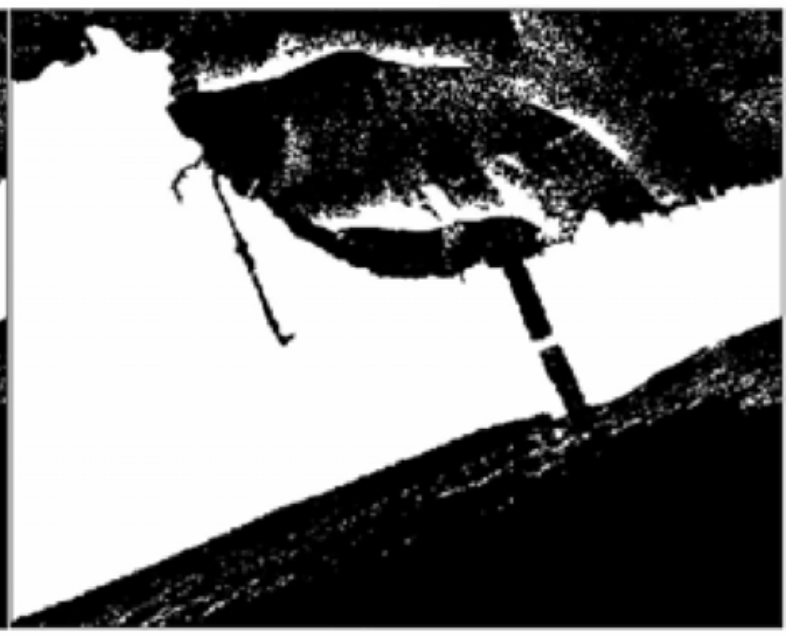

(d)

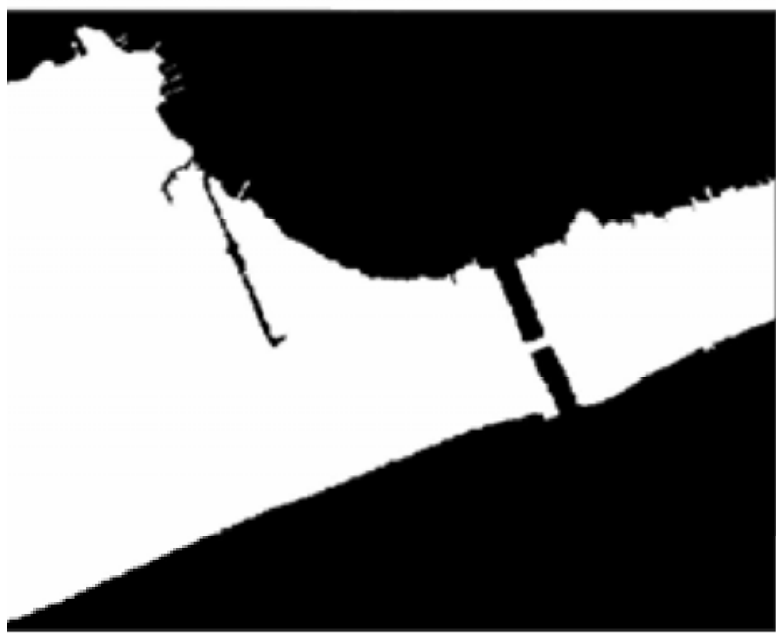

(e)

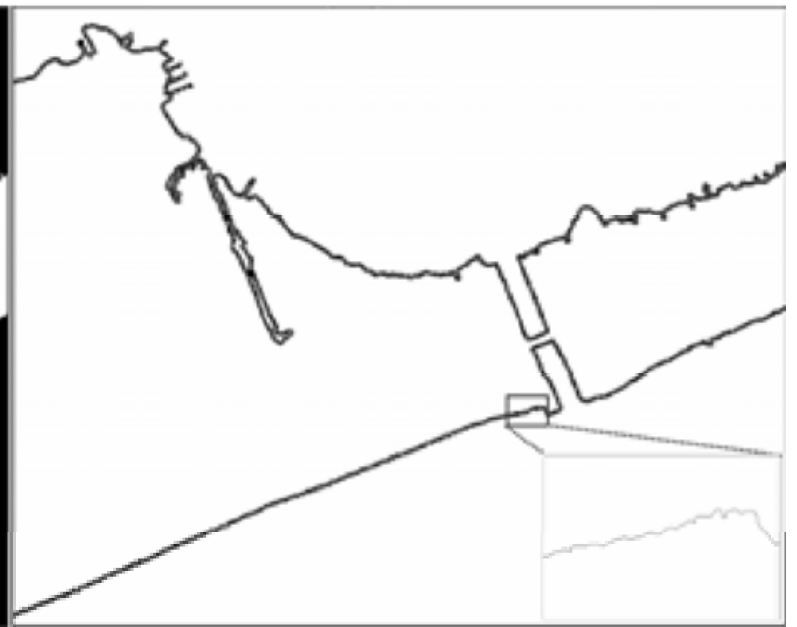

(f)

Figure 7. Shoreline extraction from LiDAR using object-based approach. (a) original LiDAR DEM; (b) LiDAR DEM after applying median filter; (c) LiDAR/tidal datum intersection result; (d) After removal of small and noisy land objects with a threshold value of $\mathbf{3 0 0 0}$ pixels; (e) After removal of small and noisy water objects with a threshold value of 4000 pixels; (f) Derived MHW shorelines. 


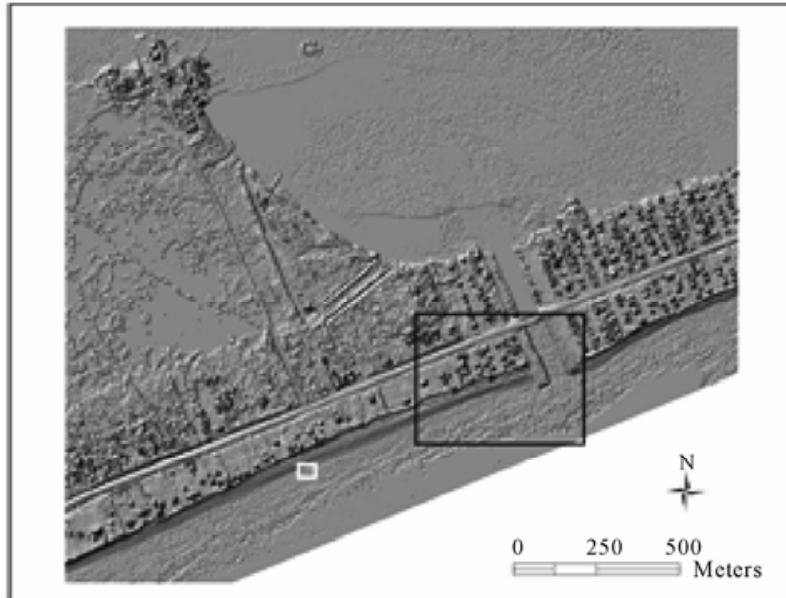

(a)

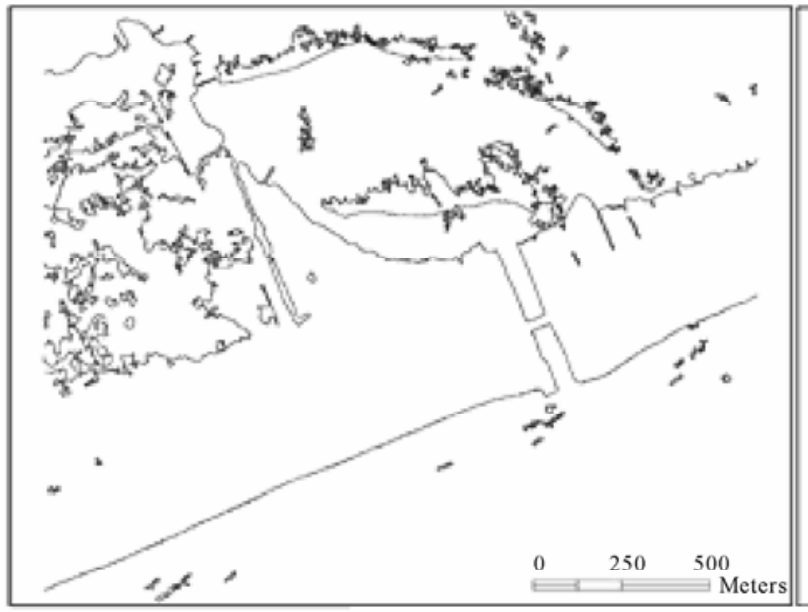

(c)

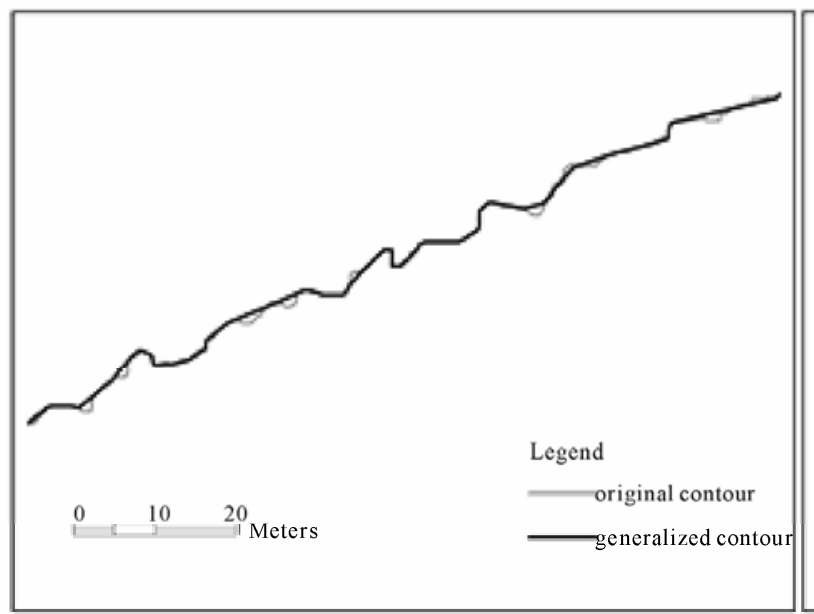

(e)

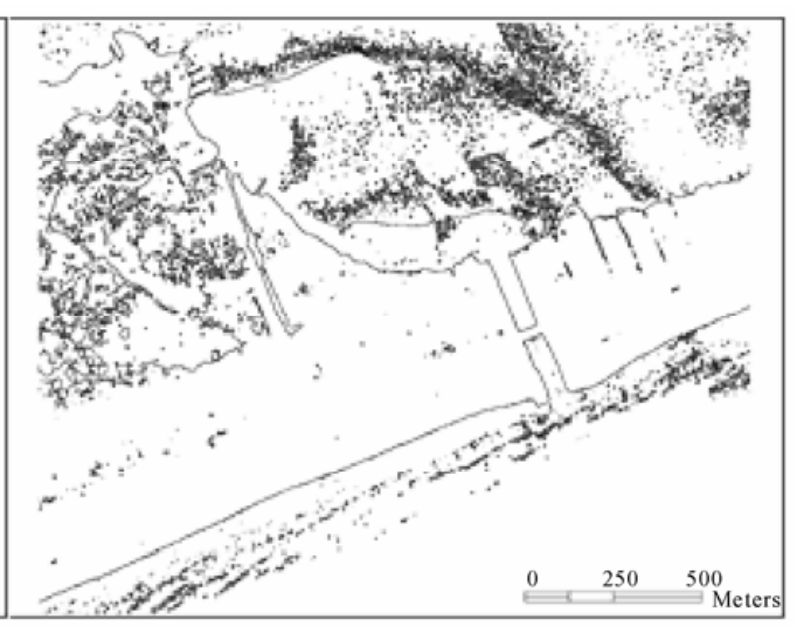

(b)

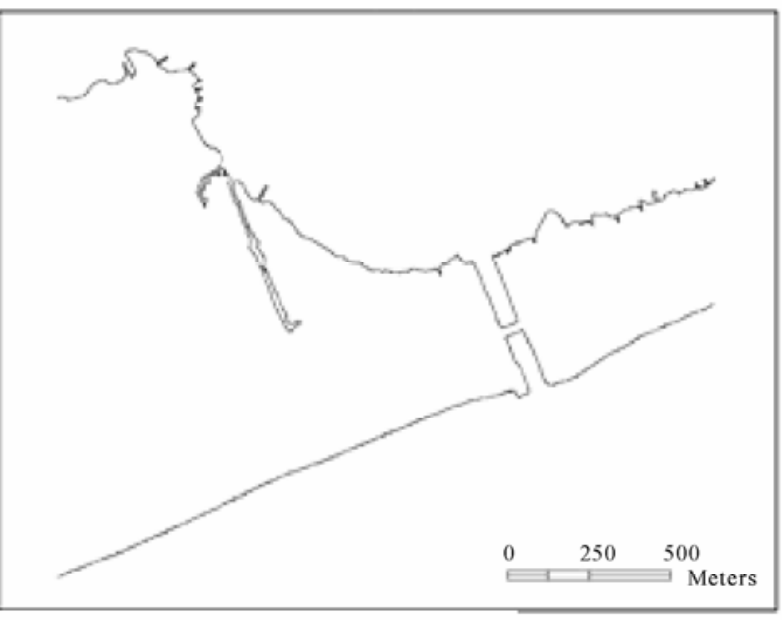

(d)

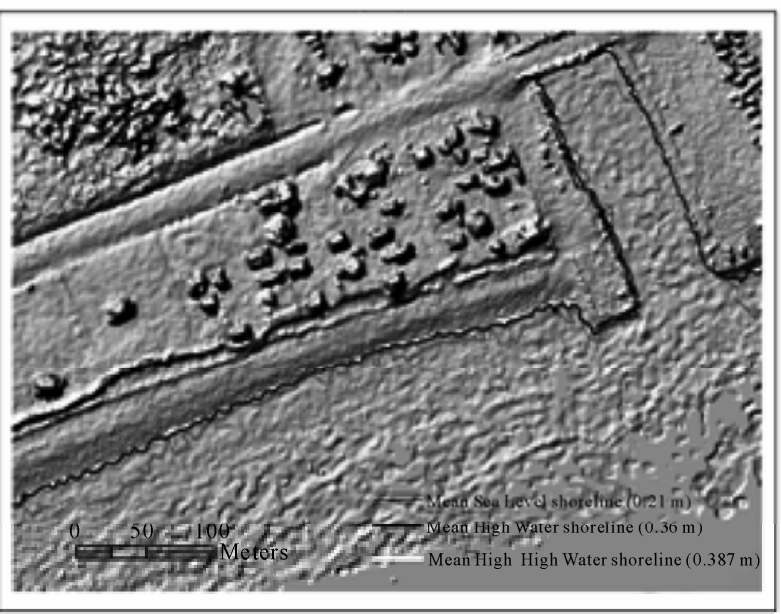

(f)

Figure 8. Shoreline extraction from LiDAR using contouring approach. (a) Hill-shaded LiDAR DEM; (b) MHW shorelines from contouring without a length threshold; (c) MHW shorelines from contouring with a length threshold of $100 \mathrm{~m}$; (d) MHW shorelines from contouring with a length threshold of $1000 \mathrm{~m}$; (e) Generalized shoreline with bend simplification option for the area in white box in A; (f) Three sets of shorelines respectively referenced to MHHW, MHW and MSL tidal datums for the area in black box of (a). 
routines under various shoreline processing scenarios. The customized graphical interfaces for all the software routines allow the user to specify and change relevant parameters. The adjustment of these parameter values can be useful for tuning the tool to a particular coastal environment or to a particular type of data source. As we demonstrated, the use of our shoreline extraction software tool can avoid the tedious and labor-intensive on-screen delineation, while producing shorelines with high precision, approaching the spatial resolution of source images or LiDAR data. It should be pointed out that our software package can be also used to extract river channels from remote sensing imagery with an appropriate selection of parameters for software routines. To achieve shoreline products with a high absolute positional accuracy, the following conditions are also required: the high spatial resolution data, sufficient contrast between land features and water bodies, the geometric integrity of the image, and accurate georeferencing and rectification of input images and LiDAR DEMs.

Our software development experiences show that the strategy of implementing and integrating the shoreline extraction routines as a plug-in extension module in the ArcGIS environment has two major advantages. First, many core GIS functions offered by the ArcObjects can be directly utilized to facilitate the development of the software routines. Secondly, embedding the shoreline extraction routines into the ArcGIS allows the user to take advantages of a wide spectrum of data management, visualization and spatial analysis capabilities during the shoreline extraction and quality control process. For a large shoreline mapping project, shorelines derived with the automated approach unavoidably contain some errors. These errors can be easily detected and corrected with the visualization and editing tools of the ArcGIS software. Our key algorithms for shoreline extraction have been implemented using the computationally high performance $\mathrm{C}++$ programming language as a series of re-usable DLLs. Besides ArcGIS, these re-usable algorithm components would be useful in the open source GIS domain like GRASS and can be optionally incorporated into other commercial software packages like the remote sensing software ENVI as a plug-in component.

\section{Acknowledgements}

This research was conducted with support from the NOAA Sea Grant Program \#NA16RG1078 and Norman Hackerman Advanced Research Program. The authors want to thank Yige Gao, and Songgang Gu for their generous assistance in testing the software routines and preparing the figures for application examples.

\section{References}

[1] D. Graham, M. Sault and J. Bailey, "National Ocean Service Shoreline - Past, Present, and Future,” Journal of Coastal Research, Vol. 38, 2003, pp. 14-32.

[2] M. Szmytkiewicz, J. X. Biegowski, L. M. Kaczmarek, T. Okrój, R. X. Ostrowski, Z. Pruszak, G. Rózyńsky and M. Skaja, "Coastline Changes nearby Harbour Structures: Comparative Analysis of One-Line Models Versus Field Data," Coastal Engineering, Vol. 40, No. 2, 2000, pp. 119-139. doi:10.1016/S0378-3839(00)00008-9

[3] D. Bellomo, M. J. Pajak and J. Sparks, "Coastal Flood Hazards and the National Flood Insurance Program," Journal of Coastal Research, Vol. 28, 1999, pp. 21-26.

[4] R. A. Morton and F. M. Speed, "Evaluation of Shorelines and Legal Boundaries Controlled by Water Levels on Sandy Beaches," Journal of Coastal Research, Vol. 14, No. 4, 1998, pp. 1373-1384.

[5] A. W. S. Smith and L. A. Jackson, "The Variability in Width of the Visible Beach,” Shore and Beach, Vol. 602, 1992, pp. 7-14.

[6] R. J. Nicholls and N. Mimura, "Regional Issues Raised by Sea-Level Rise and Their Policy Implications," Climate Research, Vol. 11, No. 1, 1999, pp. 5-18.

[7] I. Eliot, C. Finlayson and P. Waterman, "Predicted Climate Change, Sea-Level Rise and Wetland Management in the Australian Wet-Dry Tropics," Wetlands Ecology and Management, Vol. 7, No. 1, 1999, pp. 63-81. doi:10.1023/A:1008477110382

[8] R. B. Zeidler, "Continental Shorelines: Climate Change and Integrated Coastal Management,” Ocean and Coastal Management, Vol. 37, No. 1, 1997, pp. 41-62. doi:10.1016/S0964-5691(97)87434-0

[9] R. J. Nicholls and F. M. J. Hoozemans, "The Mediterranean: Vulnerability to Coastal Implications of Climate Change,” Ocean \& Coastal Management, Vol. 31, 1996, pp. 105-132. doi:10.1016/S0964-5691(96)00037-3

[10] R. J. Nicholls, N. Mimura and J. C. Topping, "Climate Change in South and South-East Asia: Some Implications for Coastal Areas,” Journal of Global Environment Engineering, Vol. 1, 1995, pp. 137-154.

[11] F. Shepard and H. Wanless, "Our Changing Coastlines,” McGraw-Hill, New York, 1971.

[12] R. Dolan, B. Hayden and S. May, "Erosion of the US Shorelines," CRC Handbook of Coastal Processes and Erosion, 1983, pp. 285-299.

[13] S. P. Leatherman and F. J. Anders, "Mapping and Managing Coastal Erosion Hazards in New York," Journal of Coastal Research Special Issue, Vol. 28, 1999, pp. 34-42.

[14] R. A. Morton, T. Miller and L. Moore, "Historical Shoreline Changes along the US Gulf of Mexico: A Summary of Recent Shoreline Comparisons and Analyses,” Journal of Coastal Research, Vol. 21, No. 4, 2005, pp. 704-709. doi:10.2112/04-0230.1

[15] K. Zhang, W. Huang, B. C. Douglas and S. P. Leatherman, "Shoreline Position Variability and Long-Term 
Trend Analysis,” Shore \& Beach, Vol. 70, No. 2, 2002, pp. 31-35.

[16] J. A. G. Cooper and O. H. Pilkey, "Sea-Level Rise and Shoreline Retreat: Time to Abandon the Bruun Rule," Global and Planetary Change, Vol. 43, No. 3-4, 2004, pp. 157-171. doi:10.1016/j.gloplacha.2004.07.001

[17] R. Tol, R. Klein and R. Nicholls, "Towards Successful Adaptation to Sea-Level Rise along Europe's Coasts,” Journal of Coastal Research, Vol. 24, 2008, pp. 432-442. doi:10.2112/07A-0016.1

[18] S. P. Leatherman, "Shoreline Mapping: A Comparison of Techniques,” Shore and Beach, Vol. 51, No. 3, 1983, pp. 28-33.

[19] R. Li, K. Di and R. Ma, "3-D Shoreline Extraction from IKONOS Satellite Imagery,” Marine Geodesy, Vol. 26, No. 1-2, 2003, pp. 107-115. doi:10.1080/01490410306699

[20] J. C. Gibeaut, W. A. White, T. Hepner, R. Gutierrez, T. A. Tremblay, R. Smyth and J. Andrews, "Texas Shoreline Change Project; Gulf of Mexico Shoreline Change from the Brazos River to Pass Cavallo,” A Report of the Texas Coastal Coordination Council, 2000.

[21] W. Robertson, D. Whitman, K. Zhang and S. P. Leatherman, "Mapping Shoreline Position Using Airborne Laser Altimetry,” Journal of Coastal Research, Vol. 20, No. 3, 2004, pp. 884-892. doi:10.2112/1551-5036(2004)20[884:MSPUAL]2.0.CO;2

[22] H. Liu, D. Sherman and S. Gu, "Automated Extraction of Shorelines from Airborne Light Detection and Ranging Data and Accuracy Assessment Based on Monte Carlo Simulation,” Journal of Coastal Research, Vol. 23, No. 6, 2007, pp. 1359-1369. doi:10.2112/05-0580.1

[23] H. Liu, "Shoreline Mapping and Coastal Change Studies Using Renote Sensing Imagery and LIDAR Data," Remote Sensing and Geospatial Technologies for Coastal Ecosystem Assessment and Management, 2009, pp. 297-322.

[24] J.-S. Lee and I. Jurkevich, "Coastline Detection and Tracing in SAR Images," IEEE Transactions on Geoscience and Remote Sensing, Vol. 28, No. 4, 1990, pp. 662-668.

[25] T. W. Ryan, P. J. Sementilli, P. Yuen and B. R. Hunt, "Extraction of Shoreline Features by Neural Nets and Image Processing," Photogrammetric Engineering \& Remote Sensing, Vol. 57, No. 7, 1991, pp. 947-955.

[26] D. C. Mason and L. J. Davenport, “Accurate and Efficient Determination of the Shoreline in ERS-1 SAR Images," IEEE Transactions on Geoscience and Remote Sensing, Vol. 34, No. 5, 1996, pp. 1243-1253. doi:10.1109/36.536540

[27] H. Liu and K. C. Jezek, “A Complete High-Resolution Coastline of Antarctica Extracted from Orthorectified Radarsat SAR Imagery," Photogrammetric Engineering and Remote Sensing, Vol. 70, No. 5, 2004, pp. 605-616.

[28] H. Liu and K. C. Jezek, “Automated Extraction of Coastline from Satellite Imagery by Integrating Canny Edge Detection and Locally Adaptive Thresholding Methods,"
International Journal of Remote Sensing, Vol. 25, No. 5, 2004, pp. 937-958. doi:10.1080/0143116031000139890

[29] H. F. Stockdon, A. H. Sallenger Jr, J. H. List and R. A. Holman, "Estimation of Shoreline Position and Change Using Airborne Topographic Lidar Data,” Journal of Coastal Research, Vol. 18, No. 3, 2002, pp. 502-513.

[30] B. B. Parker, "The Difficulties in Measuring a Consistently Defined Shoreline - the Problem of Vertical Referencing,” Journal of Coastal Research, Vol. 38, 2003, pp. 44-56.

[31] R. Smyth, J. Gibeaut, J. Andrews, T. Hepner and R. Gutierrez, "The Texas Shoreline Change Project: Coastal Mapping of West and East Bays in the Galveston Bay System Using Airborne LIDAR, Prepared for the Texas General Land Office, GLO Contract,” 2003.

[32] K. Kim, K. C. Jezek and H. Liu, "Orthorectified Image Mosaic of Antarctica from 1963 Argon Satellite Photography: Image Processing and Glaciological Applications,” International Journal of Remote Sensing, Vol. 28, No. 23, 2007, pp. 5357-5373. doi:10.1080/01431160601105850

[33] M. Sonka, V. Hlavac and R. Boyle, "Image Processing, Analysis, and Machine Vision Second Edition,” International Thomson, 1999.

[34] J. S. Lee, "Digital Image Smoothing and the Sigma Filter," Computer Vision, Graphics and Image Processing, Vol. 24, No. 2, 1983, pp. 255-269. doi:10.1016/0734-189X(83)90047-6

[35] P. Perona and J. Malik, "Scale-Space and Edge Detection Using Anisotropic Diffusion,” IEEE Transactions on Pattern Analysis and Machine Intelligence, Vol. 12, No. 7, 1990, pp. 629-639. doi:10.1109/34.56205

[36] C. K. Chow and T. Kaneko, "Automatic Boundary Detection of the Left Ventricle from Cineangiograms," Computers and Biomedical Research, Vol. 5, No. 4, 1972, pp. 388-410. doi:10.1016/0010-4809(72)90070-5

[37] R. Dubes and A. K. Jain, "Clustering Techniques: The User's Dilemma,” Pattern Recognition, Vol. 8, No. 4, 1976, pp. 247-260. doi:10.1016/0031-3203(76)90045-5

[38] J. Serra, "Image Analysis and Mathematical Morphology,” Academic Press Inc., Orlando, 1983.

[39] P. Soille, "Morphological Image Analysis: Principles and Applications,” Springer-Verlag New York, Inc., Secaucus, NJ, 2003.

[40] M. J. Pajak and S. Leatherman, "The High Water Line as Shoreline Indicator,” Journal of Coastal Research, Vol. 18, No. 2, 2002, pp. 329-337.

[41] S. A. White and Y. Wang, "Utilizing DEMs Derived from LIDAR Data to Analyze Morphologic Change in the North Carolina Coastline," Remote Sensing of Environment, Vol. 85, No. 1, 2003, pp. 39-47. doi:10.1016/S0034-4257(02)00185-2

[42] K. Zhang, D. Whitman, S. Leatherman and W. Robertson, "Quantification of Beach Changes Caused by Hurricane Floyd along Florida's Atlantic Coast Using Airborne Laser Surveys,” Journal of Coastal Research, Vol. 21, No. 1, 2005, pp. 123-134. doi:10.2112/02057.1 
[43] C. W. Finkl, L. Benedet and J. L. Andrews, "Interpretation of Seabed Geomorphology Based on Spatial Analysis of High-Density Airborne Laser Bathymetry,” Journal of Coastal Research, Vol. 21, No. 3, 2005, pp. 501-514. doi:10.2112/05-756A.1

[44] H. Liu, L. Wang, D. Sherman, Y. Gao and Q. Wu, “An Object-Based Conceptual Framework and Computational Method for Representing and Analyzing Coastal Morphological Changes," International Journal of Geographical Information Science, Vol. 24, No. 7, 2010, pp. 1015- 1041. doi:10.1080/13658810903270569
[45] K. W. Hess, "Tidal Datums and Tide Coordination," Journal of Coastal Research, Vol. 38, 2003, pp. 33-43.

[46] R. A. Morton, "Accurate Shoreline Mapping. Past, Present, and Future," Proceedings a Specialty Conference on Quantitative Approaches to Coastal Sediment Processes, Seattle, WA, USA, June 1991, pp. 997-1010.

[47] S. P. Leatherman, B. C. Douglas and J. L. LaBrecque, "Sea Level and Coastal Erosion Require Large-Scale Monitoring,” Eos, Vol. 84, No. 2, Januray 2003. 